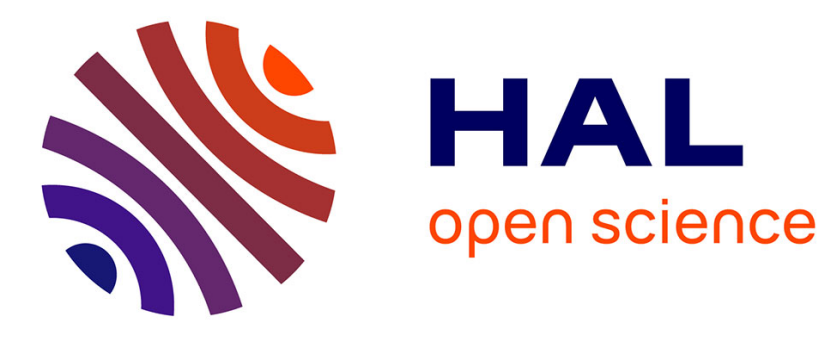

\title{
An analytical model for porous single crystals with ellipsoidal voids
}

Armel Mbiakop, Andrei Constantinescu, Kostas Danas

\section{To cite this version:}

Armel Mbiakop, Andrei Constantinescu, Kostas Danas. An analytical model for porous single crystals with ellipsoidal voids. Journal of the Mechanics and Physics of Solids, 2015, 84, pp.436-467. 10.1016/j.jmps.2015.07.011 . hal-01219387

\section{HAL Id: hal-01219387 \\ https://hal-polytechnique.archives-ouvertes.fr/hal-01219387}

Submitted on 21 Dec 2017

HAL is a multi-disciplinary open access archive for the deposit and dissemination of scientific research documents, whether they are published or not. The documents may come from teaching and research institutions in France or abroad, or from public or private research centers.
L'archive ouverte pluridisciplinaire HAL, est destinée au dépôt et à la diffusion de documents scientifiques de niveau recherche, publiés ou non, émanant des établissements d'enseignement et de recherche français ou étrangers, des laboratoires publics ou privés. 


\section{Author's Accepted Manuscript}

An analytical model for porous single crystals with ellipsoidal voids

A. Mbiakop, A. Constantinescu, K. Danas

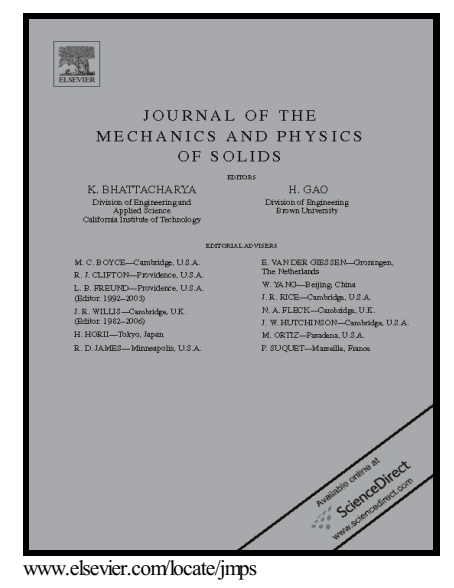

PII: $\quad$ S0022-5096(15)30017-X

DOI: $\quad$ http://dx.doi.org/10.1016/j.jmps.2015.07.011

Reference: MPS2689

To appear in: Journal of the Mechanics and Physics of Solids

Received date: 8 April 2015

Revised date: 8 July 2015

Accepted date: 12 July 2015

Cite this article as: A. Mbiakop, A. Constantinescu and K. Danas, An analytical model for porous single crystals with ellipsoidal voids, Journal of the Mechanics and Physics of Solids, http://dx.doi.org/10.1016/j.jmps.2015.07.011

This is a PDF file of an unedited manuscript that has been accepted for publication. As a service to our customers we are providing this early version of the manuscript. The manuscript will undergo copyediting, typesetting, and review of the resulting galley proof before it is published in its final citable form. Please note that during the production process errors may be discovered which could affect the content, and all legal disclaimers that apply to the journal pertain. 


\title{
An analytical model for porous single crystals with ellipsoidal voids
}

\author{
A. Mbiakop ${ }^{\mathrm{a}}$, A. Constantinescu ${ }^{\mathrm{a}}, \mathrm{K}$. Danas $^{\mathrm{a}, *}$ \\ ${ }^{a}$ Laboratoire de Mécanique des Solides, C.N.R.S. UMR7649 8 Département de Mécanique, \\ École Polytechnique, ParisTech, 91128 Palaiseau Cedex, France
}

\begin{abstract}
A rate-(in)dependent constitutive model for porous single crystals with arbitrary crystal anisotropy (e.g., FCC, BCC, HCP etc) containing general ellipsoidal voids is developed. The proposed model, denoted as modified variational model (MVAR), is based on the nonlinear variational homogenization method, which makes use of a linear comparison porous material to estimate the response of the nonlinear porous single crystal. Periodic multi-void finite element simulations are used in order to validate the MVAR for a large number of parameters including cubic (FCC, BCC) and hexagonal (HCP) crystal anisotropy, various creep exponents (i.e., nonlinearity), several stress triaxiality ratios, general void shapes and orientations and various porosity levels. The MVAR model, which involves a priori no calibration parameters, is found to be in good agreement with the finite element results for all cases considered in the rate-dependent context. The model is then used in a predictive manner to investigate the complex response of porous single crystals in several cases with strong coupling between the anisotropy of the crystal and the (morphological) anisotropy induced by the shape and orientation of the voids. Finally, a simple way of calibrating the MVAR with just two adjustable parameters is depicted in the rate-independent context so that an excellent agreement with the FE simulation results is obtained. In this last case, this proposed model can be thought as a generalization of the Gurson model in the context of porous single crystals and general ellipsoidal void shapes and orientations.
\end{abstract}

Key words: Crystal plasticity, Porous materials, Homogenization

\section{Introduction}

The constitutive response of metallic alloys is strongly affected by voids originating in the manufacturing process, that have an important effect on the lifetime as well as deformability of materials. Indeed, the growth of initially present processing induced voids in a nickel based single crystal superalloy as well as in standard polycrystals played a significant role in limiting creep life, as recently shown by experimental observations (Srivastava et al., 2012; Kondori and Benzerga, 2014b,a) at high enough temperatures on tensile specimens. The presence of voids (or cracks) in metals is known to be one of the major causes of ductile failure, as addressed in earlier works by Mc Clintock (1968), Rice and Tracey (1969) and Gurson (1977). So far, a large majority of the studies have been carried out in the context of two-phase material systems comprising an isotropic rate-(in)dependent matrix phase (metal usually described by von Mises yield criterion or creep potential) and a voided phase (pores of spherical, spheroidal or arbitrary ellipsoidal shapes). The bibliography on this subject is vast and the reader is referred to a recent review of Benzerga and Leblond (2010) for more details on the subject.

For rate-(in)dependent anisotropic matrix systems, fewer results have been obtained, generally based on a phenomenological Hill-type matrix (see Benzerga et al. (2004); Monchiet et al. (2008); Keralavarma et al. (2011)). Porous single crystals have for instance been studied through discrete dislocations dynamics (Huang et al., 2007; Hussein et al., 2008; Segurado and Llorca, 2010; Huang et al., 2012), molecular dynamics at smaller scales (Traiviratana et al., 2008; Zhao et al., 2009; Tang et al., 2010a,b), finite element simulations (Yerra et al., 2010; Ha and Kim, 2010), as well as other techniques such as quasi-continuum methods (Sorkin et al., 2014; Amelang et al., 2015) and coarse-grained atomistic models (Shenoy et al.,

\footnotetext{
* Corresponding author.

Email addresses: mbiakop@lms.polytechnique.fr (A. Mbiakop), andrei.constantinescu@lms.polytechnique.fr (A. Constantinescu), kdanas@lms.polytechnique.fr (K. Danas)
} 


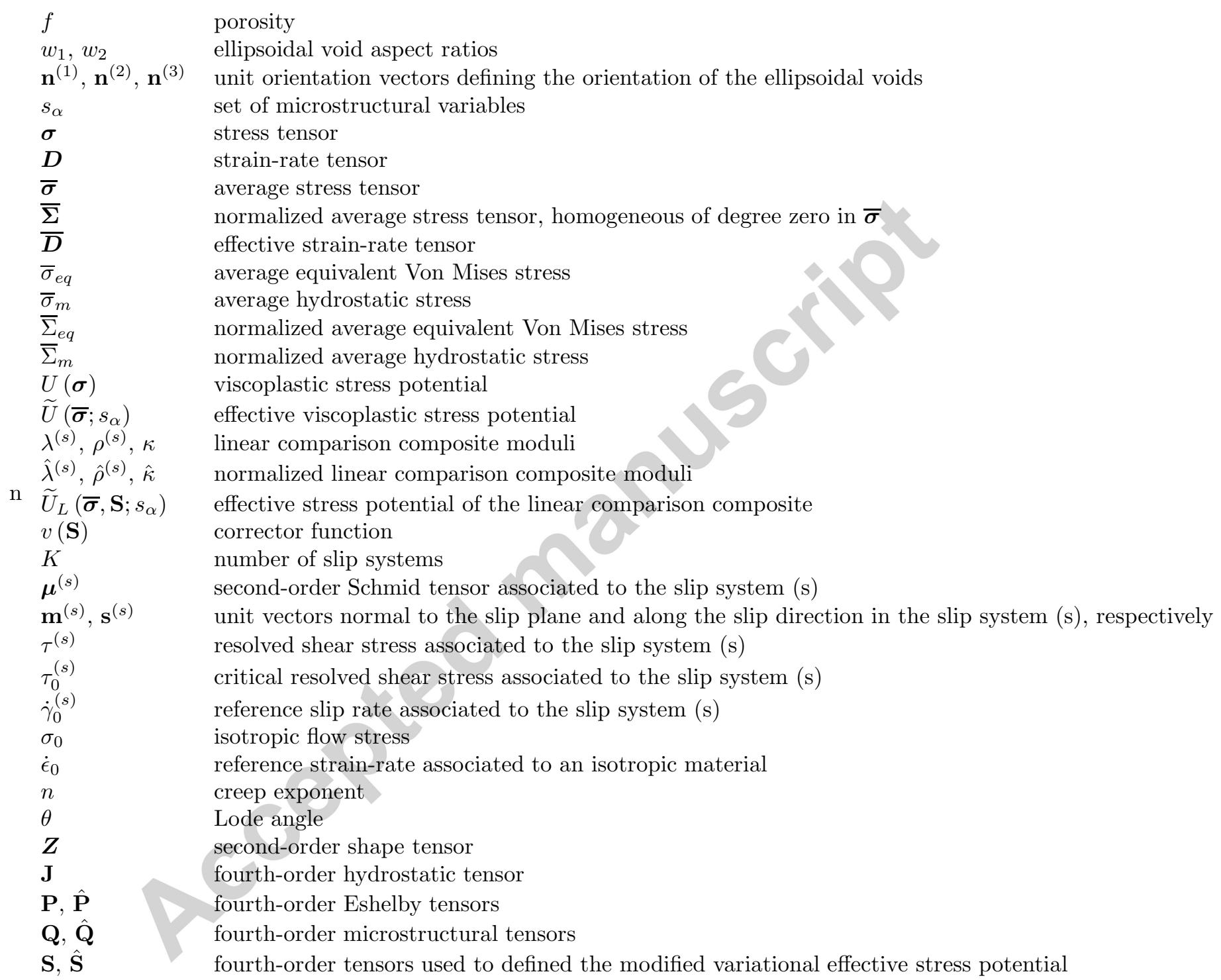


2000; Marshall and Dayal, 2014). Such anisotropic matrix systems have known slip directions and contain usually a small volume fraction of impurities. When these material systems are subjected to external loads impurities fracture or decohere leading to the creation of voids of various shapes, which in turn evolve in size, shape and orientation during the deformation process (Srivastava and Needleman, 2012). This complex evolution of microstructure together with the evolution of the rate-dependent matrix anisotropy is critical in the prediction of the eventual failure of the specimen under monotonic and cyclic loading conditions.

Nonetheless, there have been only a handful of models for porous single crystals which deal with special void geometries, loading conditions and slip system orientations. Such studies involve the study of cylindrical voids with circular cross-section in a rigid-ideally plastic face-centered cubic (FCC) single crystals using slip line theory (Kysar et al., 2005; Gan et al., 2006; Gan and Kysar, 2007), the study of two-dimensional "out of plane" cylindrical voids with circular cross-section subjected to anti-plane loadings (Idiart and Ponte Castañeda, 2007) and that of spherical voids and general loading conditions (Han et al., 2013; Paux et al., 2015). While each one of these studies has its own significant contribution to the understanding of the effective response of porous single crystals none of them is general enough in the sense of arbitrary void shapes and orientations and general loading conditions.

The scope of the present work is therefore to develop a three-dimensional model that is able to deal with arbitrary crystal anisotropy, arbitrary ellipsoidal void shapes at any given orientation and general loading conditions. The proposed model is based on appropriate extension of a former work of the authors (Mbiakop et al., 2015b) in two-dimensional porous single crystals. More specifically, in Sections 2 and 3 , we use the variational linear comparison composite theory of Ponte Castañeda (1991a) to provide a fully analytical model, called the modified variational (MVAR) model (see Danas and Aravas (2012); Mbiakop et al. (2015b)), in three-dimensions. Subsequently, in Section 4, we present in detail the finite element (FE) periodic unit-cell framework which will be used to assess the MVAR model as well as to visualize the underlying deformation fields in the context of porous single crystals. In Sections 5 and 6, we present comparisons between the MVAR predictions and the FE results for a wide range of crystal anisotropy (FCC, BCC, HCP), arbitrary ellipsoidal void shapes and orientations, porosities, creep exponents and general loading conditions. In the sequel, in Section 7, a simple way of calibrating the MVAR model in the rate-independent context with two adjustable parameters is discussed so that an excellent agreement with respect to the numerical results is obtained. Finally, we conclude with Section 8.

\section{Problem setting}

Consider a representative volume element (RVE) $\Omega$ of a two-phase porous single crystal with each phase occupying a sub-domain $\Omega^{(r)}(r=1,2)$. The vacuous phase is identified with phase 2 and the non-vacuous phase (i.e., single crystal matrix) is denoted as phase 1. At this point it is important to note that we make use of the hypothesis of separation of length scales which implies that the size of the voids (microstructure) is much smaller than the size of the single crystal and the variation of the loading conditions at the level of the single crystal.

\subsection{Microstructure}

In the present study, following previous work of Willis (1977), we consider a "particulate" microstructure which is a generalization of the Eshelby (1957) dilute microstructure in the nondilute regime. More specifically, we consider a "particulate" porous material (see Fig. 1) consisting of ellipsoidal voids aligned at a certain direction, whereas the distribution function, which is also taken to be ellipsoidal in shape, provides information about the distribution of the centers of the pores. For simplicity and in order to keep the number of microstructural variables low, we choose the shape and orientation of the distribution function to be identical to the shape and orientation of the voids themselves (see Danas and Ponte Castañeda (2009a)). Nevertheless, this analysis can be readily extended to distribution of a different shape and orientation than the voids (Ponte Castañeda and Willis (1995); Kailasam and Ponte Castañeda (1998); Agoras and Ponte Castañeda (2013)). Thus, as shown in Fig. 1, the internal variables characterizing the state of the microstructure are:

- the porosity or volume fraction of the voids $f=V_{2} / V$, where $V=V_{1}+V_{2}$ is the total volume, with $V_{1}$ and $V_{2}$ being the volume occupied by the matrix and the vacuous phase, respectively.

- the two aspect ratios $w_{1}=a_{3} / a_{1}, w_{2}=a_{3} / a_{2}\left(w_{3}=1\right)$ with $2 a_{i}(i=1,2,3)$ denoting the lengths of the principal axes of the representative elliptical void. 
- the orientation unit vectors $\mathbf{n}^{(i)}(i=1,2,3)$, defining an orthonormal basis set, which coincides with the principal axes of the representative ellipsoidal void.

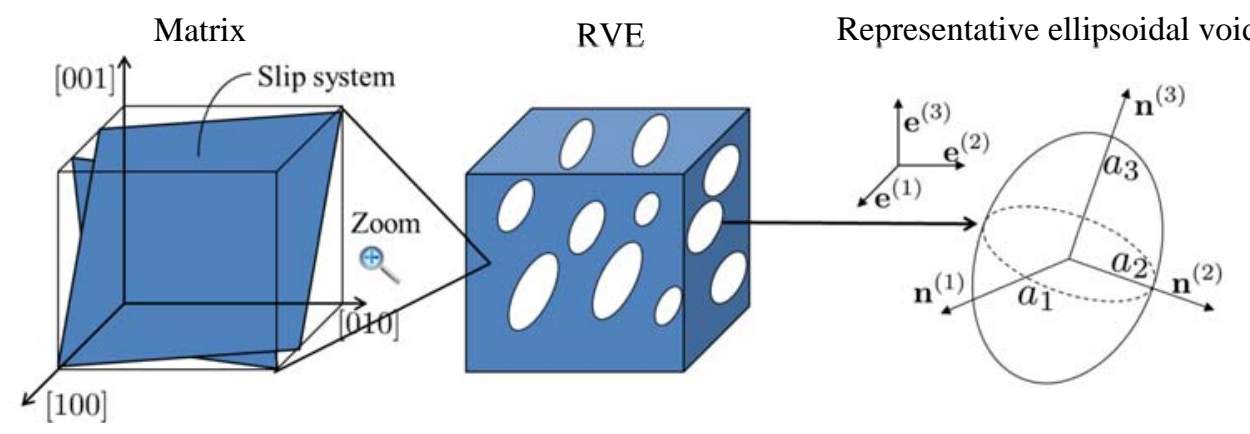

Figure 1: Representative ellipsoidal voids embedded in a crystal matrix.

The above set of the microstructural variables can then be denoted by the set

$$
s_{\alpha}=\left\{f, w_{1}, w_{2}, \mathbf{n}^{(1)}, \mathbf{n}^{(2)}, \mathbf{n}^{(3)}\right\} .
$$

Moreover, it is useful to explore other types of particulate microstructures, which can be derived easily by appropriate specialization of the aforementioned variables $s_{\alpha}$ (Budiansky et al. (1982)). In this regard, the following cases can be considered:

- $a_{1} \rightarrow \infty$ or $a_{2} \rightarrow \infty$ or $a_{3} \rightarrow \infty$. In this case, if the porosity $f$ remains finite, the cylindrical microstructure is recovered, whereas if $f \rightarrow 0$, a porous material with infinitely thin needles is generated.

$-a_{1} \rightarrow 0$ or $a_{2} \rightarrow 0$ or $a_{3} \rightarrow 0$. Here, if the porosity $f$ remains finite, a porous laminated microstructure is recovered (or alternatively a "porous sandwich"), whereas if $f \rightarrow 0$, a porous material with penny-shaped cracks is formed and thus the notion of density of cracks needs to be introduced.

To conclude, in the general case, where the aspect ratios and the orientation of the ellipsoidal voids are such that $w_{1} \neq w_{2} \neq 1$ and $\mathbf{n}^{(i)} \neq \mathbf{e}^{(i)}$, the void shape (morphological) induced anisotropy is superposed upon the existing anisotropic single crystal leading to an overall non trivial anisotropic response of the composite whose modeling is a significant challenge.

\subsection{Effective behavior: General considerations}

The local constitutive behavior of the matrix phase is characterized by an anisotropic, convex stress potential $U_{1} \equiv U$ while the stress potential of the porous phase $U_{2} \equiv 0$. As a consequence of the Hill-Mandel lemma (Hill, 1963; Mandel, 1964)), the effective stress potential $\widetilde{U}$ for a porous medium is reduced to

$$
\widetilde{U}\left(\overline{\boldsymbol{\sigma}} ; s_{\alpha}\right)=(1-f) \inf _{\boldsymbol{\sigma} \in S(\overline{\boldsymbol{\sigma}})} \frac{1}{V\left(\Omega^{(1)}\right)} \int_{\Omega^{(1)}} U(\boldsymbol{\sigma}) d V
$$

where

$$
S(\overline{\boldsymbol{\sigma}})=\left\{\boldsymbol{\sigma}, \operatorname{div}(\boldsymbol{\sigma})=0 \text { in } \Omega^{(1)}, \boldsymbol{\sigma} \mathbf{n}=0 \text { on } \partial \Omega^{(2)}, \frac{1}{V(\Omega)} \int_{\Omega} \boldsymbol{\sigma} d V=\overline{\boldsymbol{\sigma}}\right\},
$$

is the set of statically admissible stresses that are compatible with the average stress $\overline{\boldsymbol{\sigma}}$ and a traction free void surface.

Subsequently, the effective strain-rate tensor can be expresses as

$$
\overline{\boldsymbol{D}}=\frac{\partial \widetilde{U}}{\partial \overline{\boldsymbol{\sigma}}}(\boldsymbol{\sigma})
$$

In general, one has to deal with nonlinear constitutive relations for the constituents as well as random spatial distributions of the voids and consequently, the above described problem is non-trivial. In this view, the goal of the present work is to propose approximate, albeit robust and rigorous, homogenized models for such porous single crystals. In the next sections, we define the local constitutive response of the single crystal matrix and we provide both analytical and numerical estimates of the effective response of such porous single crystals. 


\subsection{Constitutive behavior of the constituents}

Let us consider a reference single crystal which undergoes viscoplastic deformation on a set of $K$ preferred crystallographic slip systems. At this stage, for simplicity in the homogenization procedure elasticity effects are neglected. These systems are characterized by the second-order Schmid tensors $\boldsymbol{\mu}^{(s)}(\forall s=1, \ldots, K)$ given by

$$
\boldsymbol{\mu}^{(s)}=\frac{1}{2}\left(\mathbf{m}^{(s)} \otimes \mathbf{s}^{(s)}+\mathbf{s}^{(s)} \otimes \mathbf{m}^{(s)}\right)
$$

with $\mathbf{m}^{(s)}$ and $\mathbf{s}^{(s)}$ denoting the unit vectors normal to the slip plane and along the slip direction in the $s^{\text {th }}$ system, respectively.

When the crystal is subjected to a stress $\boldsymbol{\sigma}$, the resolved shear stress acting on the $s^{t h}$ slip system is given by the Schmid law

$$
\tau^{(s)}=\boldsymbol{\sigma} \cdot \boldsymbol{\mu}^{(s)},
$$

while the strain-rate $\boldsymbol{D}$ in the crystal is assumed to be given by the superposition of the slip-rates of each slip system, i.e. $\boldsymbol{D}=\sum_{s=1}^{K} \dot{\gamma}^{(s)} \boldsymbol{\mu}^{(s)}$. The slip-rate $\dot{\gamma}^{(s)}$ is assumed to depend on the resolved shear stress $\tau^{(s)}$, via a slip potential $\Psi^{(s)}$ in such a way that

$$
\dot{\gamma}^{(s)}=\frac{\partial \Psi^{(s)}}{\partial \tau^{(s)}}\left(\tau^{(s)}\right) .
$$

The slip potentials $\Psi^{(s)}$ are assumed to be convex, incompressible and expressed via the power-law form

$$
\Psi^{(s)}(\tau)=\frac{\dot{\gamma}_{0}^{(s)} \tau_{0}^{(s)}}{n+1}\left(\frac{|\tau|}{\tau_{0}^{(s)}}\right)^{n+1}
$$

where $n \geq 1, \dot{\gamma}_{0}^{(s)}$ and $\tau_{0}^{(s)}$ denote the creep exponent, the reference slip-rate and the reference flow stress (also denoted as critical resolved shear stress CRSS) of the $s^{\text {th }}$ slip system, respectively. In addition, let us notice that the limiting values of the exponent, $n=1$ and $n \rightarrow \infty$ correspond to linear viscoelasticicty and rate-independent perfect plasticity, respectively. In this connection, it is recalled that, even though the slip potentials $\Psi^{(s)}$ are not differentiable in the perfect crystal plasticity case, it is still possible to relate $\dot{\gamma}^{(s)}$ and $\tau^{(s)}$ via the subdifferential of convex analysis.

An alternative and equivalent way to write down $\tau^{(s)}$ in equation (6) is by use of the fourth-order "Schmid" projection tensors $\mathbf{E}^{(s)}$ (see DeBotton and Ponte Castañeda (1995)), such that

$$
\left|\tau^{(s)}\right|=\sqrt{\frac{\boldsymbol{\sigma} \cdot \mathbf{E}^{(s)} \cdot \boldsymbol{\sigma}}{2}}, \quad \mathbf{E}^{(s)}=2 \boldsymbol{\mu}^{(s)} \otimes \boldsymbol{\mu}^{(s)} \quad \forall s=1, K .
$$

Adding up the potentials of all the slip systems we obtain the constitutive behavior of the matrix phase, characterized by the viscoplastic stress potential $U$ such that

$$
U(\boldsymbol{\sigma})=\sum_{s=1}^{K} \Psi^{(s)}\left(\tau^{(s)}\right)=\sum_{s=1}^{K} \frac{\dot{\gamma}_{0}^{(s)} \tau_{0}^{(s)}}{n+1}\left(\frac{\left|\tau^{(s)}\right|}{\tau_{0}^{(s)}}\right)^{n+1}=\sum_{s=1}^{K} \frac{\dot{\gamma}_{0}^{(s)} \tau_{0}^{(s)}}{n+1}\left(\frac{\boldsymbol{\sigma} \cdot \mathbf{E}^{(s)} \cdot \boldsymbol{\sigma}}{2\left(\tau_{0}^{(s)}\right)^{2}}\right)^{\frac{n+1}{2}}
$$

Obviously, in this last expression, $U$ is a homogeneous function of degree $n+1$ in the stress $\boldsymbol{\sigma}$.

The corresponding Cauchy stress $\boldsymbol{\sigma}$ and the Eulerian strain-rate $\boldsymbol{D}$ in the matrix are related through the constitutive relation

$$
\boldsymbol{D}=\frac{\partial U}{\partial \boldsymbol{\sigma}}(\boldsymbol{\sigma})
$$

\subsection{The limit of infinite equiangular slip systems}

The purpose of this section is to establish a connection between stress potentials corresponding to the case of infinite slip systems (i.e., $K \rightarrow \infty$ ) and purely isotropic stress potentials. The results of this section will be used in section 3.3 to obtain accurate estimates of the porous single crystal in the case of purely hydrostatic loading conditions. 
In view of this scope, we choose to study the special, albeit very useful case of $K$ equiangular slip systems (system equally partitioned in 3D space) with identical CRSS and reference slip-rate such that

$$
\tau_{0}^{(s)}=\tau_{0}, \dot{\gamma}_{0}^{(s)}=\dot{\gamma}_{0}, \quad \forall s=1, K .
$$

This analysis will be shown to be of critical importance in the following sections.

If one considers a triaxial loading state such that the three stresses are aligned with the fixed laboratory axes, the stress tensor can be written as

$$
\boldsymbol{\sigma}=\sigma_{m}\{1,1,1\}+\frac{2 \sigma_{e q}}{3}\left\{\cos \theta,-\cos \left(\theta+\frac{\pi}{3}\right),-\cos \left(\theta-\frac{\pi}{3}\right)\right\}
$$

where $\sigma_{e q}=\sqrt{3 s_{i j} s_{i j} / 2}, \sigma_{m}=\sigma_{k k} / 3$ and $\cos (\theta)=27 \operatorname{det}\left(s_{i j}\right) / 2 \sigma_{e q}^{3}$ denote the equivalent Von Mises stress, the hydrostatic stress and the Lode angle, respectively, while $\mathbf{s}$ is the stress deviator. By introducing this relation in equation (10) in the limit $K \longrightarrow \infty$, the viscoplastic stress potential $U$ becomes isotropic and its numerical computation leads to the following isotropic potential (see details in appendix A)

$$
\lim _{K \rightarrow \infty} U(\boldsymbol{\sigma})=\frac{\beta_{n}}{n+1}\left(\sigma_{e q} g_{\theta}\right)^{n+1} \lim _{K \rightarrow \infty}\left(\dot{\gamma}_{0} \tau_{0}^{-n} K\right) .
$$

Here, $g_{\theta}$ is a periodic function of $\theta$ with period $\pi / 3$ (i.e., $g_{0}=g_{\pi / 3}$ ) while $\beta_{n}$ is a monotonically decreasing function of the exponent $n$. These functions were obtained numerically (see details in appendix $\mathrm{A}$ ) and fitted with simple analytical expressions, such that

$$
g_{\theta} \cong\left(\cos \left(\theta-\frac{\pi}{3}\right)\right)^{n+1}, \quad \beta_{n} \cong \frac{4}{25} 6^{-\frac{n}{2}}
$$

Next, by introducing $\sigma_{0}$ and $\dot{\epsilon}_{0}$ as the reference strain-rate and isotropic flow stress of an isotropic material, respectively, one can set

$$
\beta_{n} \lim _{K \rightarrow \infty}\left(\dot{\gamma}_{0} \tau_{0}^{-n} K\right)=\dot{\epsilon_{0}} \sigma_{0}^{-n}
$$

This implies that the infinite-slip-system stress potential $\lim _{K \rightarrow \infty} U(\boldsymbol{\sigma})$ in equation (14) leads to a Lodedependent isotropic stress potential of the form

$$
U_{\theta}(\boldsymbol{\sigma})=\frac{\dot{\epsilon_{0}} \sigma_{0}^{-n}}{n+1}\left(\sigma_{e q} g_{\theta}\right)^{n+1}
$$

As already noted in the beginning of this section, this last result will be very useful in obtaining accurate results for the purely hydrostatic response of porous single crystals (see section 3.3) by appropriate use of exact results that exist in the context of porous materials with purely isotropic matrix phase (such as the one in equation(17)) and hydrostatic stress loading states.

\subsection{Gauge surface}

In this section, we recall the notion of the gauge surface. Gauge surfaces are equipotential surfaces which have a physical importance in the present analysis as they characterize the domain of statically admissible stresses and are analogous to the yield surfaces in rate-independent plasticity.

Using the homogeneity of the stress potential (10) for single crystal matrix together with the general definition of the effective stress behavior (2), one can show that the effective stress potential $\widetilde{U}$ is also homogeneous of degree $n+1$ in $\overline{\boldsymbol{\sigma}}$.

Therefore, it is convenient to introduce the so-called gauge surface $P_{n}$ (the subscript being used to denote the dependence on the creep exponent $n$ ) as in Leblond et al. (1994). More precisely, it is sufficient to study only one of the equi-potential surfaces $\{\overline{\boldsymbol{\sigma}}, \widetilde{U}(\overline{\boldsymbol{\sigma}})=$ const $\}$ i.e., the so-called gauge surface $P_{n}$ of the porous material. For instance, in the case of slip systems with identical CRSS $\tau_{0}$ and reference slip-rate $\dot{\gamma}_{0}$, one can define the equi-potential surfaces

$$
P_{n} \equiv\left\{\bar{\Sigma}, \widetilde{U}\left(\bar{\Sigma} ; s_{\alpha}\right)=\frac{\dot{\gamma}_{0} \tau_{0}^{-n}}{n+1}\right\}
$$


Here $\overline{\boldsymbol{\Sigma}}$ is a normalized stress tensor which is homogeneous of degree zero in $\overline{\boldsymbol{\sigma}}$. Its computation in the context of numerical homogenization is further detailed in Section 5.1. Then, the gauge function $\widetilde{\Phi}_{n}$ provides the equation for the gauge surface via the expression

$$
\overline{\boldsymbol{\Sigma}} \in P_{n} \Longleftrightarrow \widetilde{\Phi}_{n}\left(\overline{\boldsymbol{\Sigma}}, s_{\alpha}\right)=(n+1) \widetilde{U}\left(\overline{\boldsymbol{\Sigma}} ; s_{\alpha}\right)-\dot{\gamma}_{0} \tau_{0}^{-n}=0 .
$$

The subscript $n$ has been used to indicate that the gauge function depends explicitly on the creep exponent of the single crystal matrix. The above definitions of the gauge surface and gauge function are analogous to the well known yield function and yield surface in the context of rate-independant plasticity $(n \rightarrow \infty)$.

\section{The homogenization model}

The homogenized model that is derived in this section derives from the nonlinear variational (VAR) homogenization method developed by Ponte Castañeda (1991a, 2002a) (see also its interpretation as a secant method by Suquet (1995)), which is based on the construction of a linear comparison composite (LCC) with the same microstructure as the nonlinear composite. The different phases of the LCC are determined through well defined linearizations of the given nonlinear phases by making use of a suitably designed variational principle.

The basic idea behind the VAR method and consequently the choice of the LCC is to replace the optimization problem with respect to the stress field $\boldsymbol{\sigma}$ in equation (2) by an optimization problem with respect to the phases of the LCC. The advantage of this operation is that instead of trying to propose stress fields (as is done usually in standard limit analysis methods) that need to satisfy the boundary conditions and are subject to differential constraints (see equation (3) for statically admissible fields), one can use piecewise constant moduli of the linearized phases to approximate the effective response of the nonlinear composite.

In order to clarify further the above described idea and the subsequent analysis, it is useful to recall first the VAR method and some of the basic steps needed to reach to the final principles in the context of two-phase porous composites. Note that the porous composite comprises two phases. A vacuous phase with stress potential equal to zero (i.e., $U^{(2)}=0$ ) and a matrix phase with $U^{(1)} \equiv U$.

Then, following the derivations presented in Ponte Castañeda (1991a, 2002a), we start by noting that $U(\boldsymbol{\sigma})=U_{L}(\boldsymbol{\sigma})+\left[U(\boldsymbol{\sigma})-U_{L}(\boldsymbol{\sigma})\right]$ with $U_{L}$ being the stress potential of a linear material serving to describe the matrix in the LCC which is defined in the following. Then, using the fundamental inequality, $\inf _{x \in \mathcal{A}}\{f(x)+g(x)\} \geq \inf _{x \in \mathcal{A}}\{f(x)\}+\inf _{x \in \mathcal{A}}\{g(x)\}$ as well as the identity $\inf _{x \in \mathcal{A}}\{-g(x)\}=-\sup _{x \in \mathcal{A}}\{g(x)\}$, one gets

$$
\begin{aligned}
\widetilde{U}\left(\overline{\boldsymbol{\sigma}} ; s_{\alpha}\right) & =(1-f) \inf _{\boldsymbol{\sigma} \in S(\overline{\boldsymbol{\sigma}})} \frac{1}{V\left(\Omega^{(1)}\right)} \int_{\Omega^{(1)}} U(\boldsymbol{\sigma}) d V \\
& \geq \widetilde{U}_{L}\left(\overline{\boldsymbol{\sigma}}, \mathbf{S} ; s_{\alpha}\right)+(1-f) \inf _{\boldsymbol{\sigma} \in S(\overline{\boldsymbol{\sigma}})} \frac{1}{V\left(\Omega^{(1)}\right)} \int_{\Omega^{(1)}}\left[U(\boldsymbol{\sigma})-U_{L}(\boldsymbol{\sigma}, \mathbf{S})\right] d V,
\end{aligned}
$$

where $\mathbf{S}$ is the fourth-order compliance tensor (having both minor and major symmetries) of the matrix phase in the LCC and serves as a "trial tensor" with respect to which we will optimize later. In turn, $\widetilde{U}_{L}$ denotes the effective stress potential of the linear comparison composite given formally by

$$
\widetilde{U}_{L}\left(\overline{\boldsymbol{\sigma}}, \mathbf{S} ; s_{\alpha}\right):=(1-f) \inf _{\boldsymbol{\sigma} \in S(\overline{\boldsymbol{\sigma}})} \frac{1}{V\left(\Omega^{(1)}\right)} \int_{\Omega^{(1)}} U_{L}(\boldsymbol{\sigma}, \mathbf{S}) d V .
$$

For the evaluation of $\widetilde{U}_{L}$ in the above expression any linear homogenization estimate that is available in the literature can be used. If, for instance, one considers the Hashin-Shtrikman bounds then the procedure described in (20) will deliver nonlinear bounds. If, instead, one uses the self-consistent estimates for $\widetilde{U}_{L}$, then one will get estimates for the nonlinear composite $\widetilde{U}$.

Next, we observe that the final term in (20) is as difficult to optimize as the original problem and it contains the difference between the nonlinear and the linear stress potential, i.e., $U-U_{L}$. Note however that as a result of the power-law form of $U$ (see equation (10)), the last term in (20) is negative and therefore by relaxing the constraint in $\boldsymbol{\sigma} \in S(\overline{\boldsymbol{\sigma}})$ with constant stress fields, denoted for clarity as $\widehat{\boldsymbol{\sigma}}$, we 
obtain the following inequality (Ponte Castañeda, 2002a)

$$
\begin{aligned}
\inf _{\boldsymbol{\sigma} \in S(\overline{\boldsymbol{\sigma}})} \frac{1}{V\left(\Omega^{(1)}\right)} \int_{\Omega^{(1)}}\left[U(\boldsymbol{\sigma})-U_{L}(\boldsymbol{\sigma}, \mathbf{S})\right] d V & \geq \inf _{\widehat{\boldsymbol{\sigma}}}\left[U(\widehat{\boldsymbol{\sigma}})-U_{L}(\widehat{\boldsymbol{\sigma}}, \mathbf{S})\right] \\
& =-\sup _{\widehat{\boldsymbol{\sigma}}}\left[U_{L}(\widehat{\boldsymbol{\sigma}}, \mathbf{S})-U(\widehat{\boldsymbol{\sigma}})\right] \\
& =-v(\mathbf{S}) .
\end{aligned}
$$

where the functions $v(\mathbf{S})$ have been introduced for convenience.

Finally, we observe that the inequality (20) is valid for any choice of the compliance tensor $\mathbf{S}$. Thus by combining results (21) and (22) and optimizing further the effective potential $\widetilde{U}$ with respect to $\mathbf{S}$, one gets (Ponte Castañeda, 1991a)

$$
\widetilde{U}\left(\overline{\boldsymbol{\sigma}} ; s_{\alpha}\right) \geq \sup _{\mathbf{S}}\left\{\widetilde{U}_{L}\left(\overline{\boldsymbol{\sigma}}, \mathbf{S} ; s_{\alpha}\right)-(1-f) v(\mathbf{S})\right\} .
$$

At this point it should be pointed out that the goal of the present work is to derive a simple analytical model and thus no attempt to provide bounds is made from this point on. This allows for the relaxation of the above "sup" processes and their replacement by stationary operations, which lead to estimates and not bounds. The reason for this relaxation is discussed further in Appendix B and is related to the analytical tractability of the problem (see relevant discussion in the context of equations (81) and (82)). Thus, by replacing the "sup" operations in (23) and (22) with corresponding "stat" ones, one gets VAR estimates of the form (Ponte Castañeda, 2002a)

$$
\widetilde{U}\left(\overline{\boldsymbol{\sigma}} ; s_{\alpha}\right)=\operatorname{stat}_{\mathbf{S}}\left\{\widetilde{U}_{L}\left(\overline{\boldsymbol{\sigma}}, \mathbf{S} ; s_{\alpha}\right)-(1-f) \operatorname{stat}_{\widehat{\sigma}}\left[U_{L}(\widehat{\boldsymbol{\sigma}}, \mathbf{S})-U(\widehat{\boldsymbol{\sigma}})\right]\right\} .
$$

The above operations, albeit non-trivial algebraically, are fully defined and one can proceed with the estimation of $\widetilde{U}$. We only need to define the compliance tensor, $\mathbf{S}$, of the linearized matrix phase, i.e., choose the constitutive response of the matrix in the linear comparison composite (LCC) and then carry out the above prescribed optimization operations.

\subsection{Choice of the linear comparison composite $(L C C)$}

In this section, we choose the compliance tensor $\mathbf{S}$ of the matrix phase in the linear comparison composite (LCC) in such a way that will allow us to get explicit estimates for $\widetilde{U}(\overline{\boldsymbol{\sigma}})$ in (24). The difficulty in the choice of $\mathbf{S}$ is twofold. First, $\mathbf{S}$ does not need to be constant per phase (see Herve and Zaoui (1993)). While such a choice would lead to more accurate estimates, in general, one would have to resolve to fully numerical optimization of the problem. This makes it implicit and intractable for numerical implementations and modeling of real geometries. The second point is that one can choose $\mathbf{S}$ to be piecewise constant but in its most general form, i.e, optimize with respect to all components of the fourth-order tensor $\mathbf{S}$ after symmetries are taken into account (see DeBotton and Ponte Castañeda (1995); Idiart and Ponte Castañeda (2007)). This choice again leads to a convex but sub-differentiable optimization problem for $\widetilde{U}(\overline{\boldsymbol{\sigma}})$ as a function of $\mathbf{S}$ and thus one has to resolve again to a fully numerical optimization problem (except in special cases as in Idiart and Ponte Castañeda (2007)). For this reason, in the present work, we proceed by choosing not only a piecewise constant $\mathbf{S}$, but also of a special form. This form is directly inspired by the viscoplastic stress potential of the matrix $U$ in the special linear case of $n=1$ in equation (10). Then, one can write (DeBotton and Ponte Castañeda, 1995; Mbiakop et al., 2015b)

$$
U_{L}(\boldsymbol{\sigma}, \mathbf{S})=\frac{1}{2} \boldsymbol{\sigma}(\mathbf{x}) \cdot \mathbf{S} \cdot \boldsymbol{\sigma}(\mathbf{x}), \quad \mathbf{S}=\sum_{s=1}^{K} \frac{1}{2 \lambda^{(s)}} \mathbf{E}^{(s)}+\frac{1}{2 \rho} \sum_{s=1}^{K} \mathbf{F}^{(s)}+\frac{1}{3 \kappa} \mathbf{J}, \forall s=1, K .
$$

Here, $\mathbf{S}$ has both major and minor symmetries, $\mathbf{E}^{(s)}$ are the Schmid fourth-order projection tensors defined in equation (9), $\mathbf{K}$ and $\mathbf{J}$ denote the fourth-order shear and hydrostatic projection tensors ${ }^{1}$,

\footnotetext{
${ }^{1}$ The projection tensors in index notation become, $I_{i j k l}=\left(\delta_{i k} \delta_{j l}+\delta_{i l} \delta_{j k}\right) / 2, J_{i j k l}=\delta_{i j} \delta_{k l} / 3$ and $K_{i j k l}=I_{i j k l}-J_{i j k l}$ with $i, j, k, l=1,2,3$.
} 
respectively, while $\mathbf{F}^{(s)}=\mathbf{K}-\mathbf{E}^{(s)}$. Note that for each slip system $s=1, K$, the $\operatorname{triplet}\left(\mathbf{E}^{(s)}, \mathbf{F}^{(s)}, \mathbf{J}\right)$ forms a fourth-order tensor basis ${ }^{2}$.

In the same equation $(25), \lambda^{(s)}$ are unknown moduli that remain to be optimized according to the optimization procedure in (24). By contrast, $\rho$ describes physically the modulus "perpendicular" to the slip directions. This modulus is infinite since no slip occurs in that direction and hence one can set $\rho \rightarrow \infty$. In turn, $\kappa$ is the bulk modulus which also needs to be set to $\kappa \rightarrow \infty$ due to incompressibility of the matrix phase. It is straightforward to observe that if one sets $\rho \rightarrow \infty$ and $\kappa \rightarrow \infty$ in (25), we recover the linear stress potential of equation (10). Nevertheless, these two moduli $\rho$ and $\kappa$ have been introduced so that we can evaluate the Eshelby integrals (see equations (31) and (33) below), which otherwise would have been singular. Instead by considering the limits $\rho \rightarrow \infty$ and $\kappa \rightarrow \infty$ during the evaluation of the Eshelby integrals but not before, the singularities disappear. A similar notion has already been used in the context of isotropic porous materials with the use of a bulk modulus which is set to infinity during the evaluation of the Eshelby integrals but not before (see for instance, Willis (1978) and Danas et al. $(2008 b))$.

The above choice for the LCC allows to write that $U_{L}(\boldsymbol{\sigma}, \mathbf{S}) \equiv U_{L}\left(\boldsymbol{\sigma}, \lambda^{(s)}\right)$. Then, the corrector function $v(\mathbf{S}) \equiv v\left(\lambda^{(s)}\right)$, introduced in equation (22) reads

$$
v\left(\lambda^{(s)}\right)=\underset{\widehat{\boldsymbol{\sigma}}}{\operatorname{stat}}\left(U_{L}\left(\widehat{\boldsymbol{\sigma}}, \lambda^{(s)}\right)-U(\widehat{\boldsymbol{\sigma}})\right)
$$

where it is recalled that $\widehat{\boldsymbol{\sigma}}$ is a constant second-order symmetric tensor which is obtained via the "stat" operator in (26). In the following, using (25) and (26), one can show that in the context of a porous material the effective energy function (24) takes the form

$$
\widetilde{U}_{v a r}\left(\overline{\boldsymbol{\sigma}} ; s_{\alpha}\right)=\operatorname{stat}_{\lambda^{(s)} \geq 0}\left[\widetilde{U}_{L}\left(\overline{\boldsymbol{\sigma}}, \lambda^{(s)} ; s_{\alpha}\right)-(1-f) v\left(\lambda^{(s)}\right)\right]
$$

where $f$ is the porosity and $\widetilde{U}_{L}$ is the effective energy of the LCC defined formally in equation (21) and is explicitly evaluated in Appendix B. The two optimization procedures involved first in (26) with respect to the constant tensors $\widehat{\boldsymbol{\sigma}}$ and subsequently in equation (27) with respect to the moduli $\lambda^{(s)}$ can be carried out analytically, but are too cumbersome and are detailed in Appendix B.

\subsection{Variational estimate for porous single crystal}

By carrying out the optimizations described in the previous section, we show that the variational (VAR) estimate of the effective stress potential of the porous single crystal reads

$$
\widetilde{U}_{v a r}\left(\overline{\boldsymbol{\sigma}} ; s_{\alpha}\right)=(1-f)^{-n} \sum_{s=1}^{K} \frac{\dot{\gamma}_{0}^{(s)} \tau_{0}^{(s)}}{n+1}\left(\frac{\left|\widetilde{\tau}^{(s)}\right|}{\tau_{0}^{(s)}}\right)^{n+1}, \quad \widetilde{\tau}^{(s)}=\left[\left(\overline{\boldsymbol{\sigma}} \cdot \boldsymbol{\mu}^{(s)}\right)^{2}+\frac{f}{K} \overline{\boldsymbol{\sigma}} \cdot \widehat{\mathbf{S}}^{*} \cdot \overline{\boldsymbol{\sigma}}\right]^{1 / 2}
$$

or equivalently

$$
\widetilde{U}_{\text {var }}\left(\overline{\boldsymbol{\sigma}} ; s_{\alpha}\right)=(1-f)^{-n} \sum_{s=1}^{K} \frac{\dot{\gamma}_{0}^{(s)}\left(\tau_{0}^{(s)}\right)^{-n}}{n+1}\left(\overline{\boldsymbol{\sigma}} \cdot \widehat{\mathbf{S}}^{\text {var },(s)} \cdot \overline{\boldsymbol{\sigma}}\right)^{(n+1) / 2}
$$

with

$$
\widehat{\mathbf{S}}^{\operatorname{var},(s)}=\frac{1}{2} \mathbf{E}^{(s)}+\frac{f}{K} \widehat{\mathbf{S}}^{*}
$$

for all $s=1, K$. It is interesting to note that $\widetilde{U}_{v a r}$ is of the same form as the stress potential of the matrix phase in equation (10), augmented with the additional term involving the fourth-order microstructural tensor $\widehat{\mathbf{S}}^{*}$. This tensor is a function of the void shape and orientation, as well as of the number $K$ and orientation of the slip systems, i.e., $\widehat{\mathbf{S}}^{*}=\widehat{\mathbf{S}}^{*}\left(w_{1}, w_{2}, n^{(i)}, \boldsymbol{\mu}^{(s)}, K, \mathbf{J}\right)$ with $i=1,2,3$ and $s=1, K$. More specifically, $\widehat{\mathbf{S}}^{*}$ is given by

$$
\widehat{\mathbf{S}}^{*}=\widehat{\mathbf{Q}}^{-1}-\sum_{s=1}^{K} \frac{1}{2} \mathbf{E}^{(s)}, \quad \widehat{\mathbf{Q}}=\lim _{\widehat{\rho} \rightarrow \infty} \lim _{\widehat{\kappa} \rightarrow \infty}\left[\mathcal{S}_{0}^{-1}-\mathcal{S}_{0}^{-1} \widehat{\mathbf{P}} \mathcal{S}_{0}^{-1}\right]
$$

\footnotetext{
${ }^{2} \mathbf{F}^{(s)} \cdot \mathbf{F}^{(s)}=\mathbf{F}^{(s)}, \mathbf{E}^{(s)} \cdot \mathbf{E}^{(s)}=\mathbf{E}^{(s)}, \mathbf{E}^{(s)} \cdot \mathbf{F}^{(s)}=\mathbf{F}^{(s)} \cdot \mathbf{E}^{(s)}=0, \forall s=1, K$ 
where $\mathcal{S}$ is a reduced version of the $\mathbf{S}$ tensor defined in equation (25), and reads (see Appendix B for more details)

$$
\mathcal{S}=\sum_{s=1}^{K} \frac{1}{2} \mathbf{E}^{(s)}+\frac{1}{2 \widehat{\rho}} \sum_{s=1}^{K} \mathbf{F}^{(s)}+\frac{1}{3 \widehat{\kappa}} \mathbf{J}, \quad \forall s=1, K,
$$

where $\widehat{\rho} \sim \rho$ and $\widehat{\kappa} \sim \kappa$ have been introduced for convenience and are further detailed in Appendix B in the context of equations (78) and (79). In turn, $\widehat{\mathbf{P}}$ is a microstructural tensor related to the Eshelby tensor (Eshelby, 1957) and is given by (Willis, 1977)

$$
\widehat{\mathbf{P}}_{i j k l}=\frac{1}{4 \pi \operatorname{det} \boldsymbol{Z}} \int_{|\xi|=1} \frac{\left.\left(\mathcal{S}_{i a k b}^{-1} \xi_{a} \xi_{b}\right)^{-1} \xi_{j} \xi_{l}\right|_{(i j)(k l)}}{\left|\boldsymbol{Z}^{-1} \cdot \xi\right|^{3}} d S .
$$

The brackets $(i j)(k l)$ denote symmetrization with respect to the corresponding indices, $\xi$ is a unit threedimensional vector and $\boldsymbol{Z}$ is a second-order tensor serving to describe the shape and orientation of the voids, i.e.,

$$
\boldsymbol{Z}=w_{1} \mathbf{n}^{(1)} \otimes \mathbf{n}^{(1)}+w_{2} \mathbf{n}^{(2)} \otimes \mathbf{n}^{(2)}+\mathbf{n}^{(3)} \otimes \mathbf{n}^{(3)} .
$$

At present, the evaluation of the tensor $\widehat{\mathbf{S}}^{*}$, i.e., both the limits $\widehat{\rho}$ and $\widehat{\kappa}$ in equation (31) as well as the integration in (33), are carried out numerically. Further optimization of this procedure is needed particularly if extreme values of the aspect ratios (e.g., $w_{i}<0.05$ or $w_{i}>20$ with $i=1,2$ ) are used since the kernel of the integral (33) becomes highly oscillatory. Nonetheless, such an operation is beyond the scope of this study and will not be pursued here. It should be mentioned, however, that in the case of two-dimensional systems ( $2 D$ crystal plasticity and cylindrical voids), the tensor $\widehat{\mathbf{S}}^{*}$ can be evaluated analytically as discussed in Mbiakop et al. (2015b).

\subsection{Correction of the hydrostatic point}

It is well known from Ponte Castañeda (1991b) and Michel and Suquet (1992)) that in the case of isotropic matrix and hydrostatic loadings the variational estimates are overly stiff. This is also the case in the present work, i.e. the estimate $(29)$ for $\widetilde{U}_{v a r}$ is very stiff when compared to numerical unit-cell calculations (performed in the present study and described in the following section). A way to remedy this overly stiff response is to use exact results that are available in the context of porous materials with isotropic matrix phases such as those derived in equation (17).

In this regard, the goal of this section is to propose a correction to the $\widetilde{U}_{v a r}$ estimate (29) in the limit of purely hydrostatic loadings. Unfortunately, in the present case of anisotropic crystal plasticity no simple analytical solution is available in the purely hydrostatic limit. Nevertheless, one can insist that the estimate (29) must recover the result of an isotropic spherical porous shell (or equivalently a composite sphere assemblage (CSA) microstructure) in the limit of infinite equiangular slip systems $(K \rightarrow \infty)$, spherical voids and hydrostatic loadings. The reason is that in that theoretical limit, the response becomes fully isotropic, with a matrix phase described by a stress potential of the form (17).

Specifically, it has recently been shown by Benallal et al. (2014); Benallal (2015) (but see also in the context of a Tresca porous material the works of Cazacu et al. (2014); Revil-Baudard and Cazacu (2014)) that the purely hydrostatic response of a spherical porous shell whose matrix phase is described by a stress potential of the form (17) is identical to that of a porous spherical shell with a $J_{2}$-type matrix phase and is given by the closed form expression (Leblond et al., 1994; Danas et al., 2008b)

$$
\widetilde{U}_{i s o}(\overline{\boldsymbol{\sigma}})=\frac{\dot{\epsilon_{0}} \widetilde{\sigma}_{h}}{n+1}\left(\frac{3 \bar{\sigma}_{m}}{2 \widetilde{\sigma}_{h}}\right)^{n+1}, \quad \frac{\widetilde{\sigma}_{h}}{\sigma_{0}}=n\left(f^{-1 / n}-1\right)
$$

where $\sigma_{0}$ and $\dot{\epsilon}_{0}$ denote the reference strain-rate and the isotropic flow stress of the isotropic matrix. This result is valid for isotropic potentials such as the one in equation (17) corresponding to infinite slip systems.

Next, motivated by similar work on isotropic matrix systems (Danas and Aravas (2012) but see also

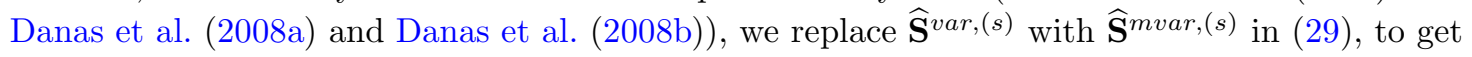

$$
\widetilde{U}_{\text {mvar }}\left(\overline{\boldsymbol{\sigma}} ; s_{\alpha}\right)=(1-f)^{-n} \sum_{s=1}^{K} \frac{\dot{\gamma}_{0}^{(s)}\left(\tau_{0}^{(s)}\right)^{-n}}{n+1}\left(\overline{\boldsymbol{\sigma}} \cdot \widehat{\mathbf{S}}^{\text {mvar },(s)} \cdot \overline{\boldsymbol{\sigma}}\right)^{(n+1) / 2},
$$


where

$$
\widehat{\mathbf{S}}^{\text {mvar },(s)}=\widehat{\mathbf{S}}^{\operatorname{var},(s)}+\left(q_{J}^{2}-1\right) \mathbf{J} \cdot \widehat{\mathbf{S}}^{\operatorname{var},(s)} \cdot \mathbf{J} .
$$

The label "mvar" refers to "modified variational" (MVAR), whereas the factor " $q_{J}$ " remains to be identified so that the MVAR estimate recovers the exact result (35) in the limit of infinite slip systems $K \longrightarrow \infty$.

In view of this, we consider a purely hydrostatic loading $\overline{\boldsymbol{\sigma}}_{i j}=\bar{\sigma}_{m} \delta_{i j}$ and spherical voids (i.e., $w_{1}=w_{2}=1$ ) embedded in a single crystal comprising slip systems with identical CRSS and reference slip rate, i.e. $\left(\tau_{0}\right)^{(s)}=\tau_{0},\left(\dot{\gamma}_{0}\right)^{(s)}=\dot{\gamma}_{0}, \forall s=1, K$. In the case of infinite equiangular slip systems and purely hydrostatic loadings, equation (36) becomes

$$
\widetilde{U}_{\text {mvar }}\left(\overline{\boldsymbol{\sigma}} ; s_{\alpha}\right) \cong(1-f)^{-n}\left(\frac{3 f}{20} q_{J}^{2}\right)^{\frac{n+1}{2}} \frac{\bar{\sigma}_{m}^{n+1}}{n+1} \lim _{K \rightarrow \infty}\left(\dot{\gamma}_{0} \tau_{0}^{-n} K\right) .
$$

Next, using equation (16), and setting equation (35) equal to (38), i.e., $\widetilde{U}_{m v a r}=\widetilde{U}_{i s o}$, we readily obtain

$$
q_{J}=\sqrt{\frac{15}{f}}\left\{\frac{(1-f)\left(\beta_{n}\right)^{\frac{1}{n}}}{n(f-1 / n-1)}\right\}^{\frac{n}{n+1}}
$$

where $\beta_{n}$ is given in (15).

While this correction establishes that the $\widetilde{U}_{\text {mvar }}$ in $(36)$ recovers the isotropic limit (i.e., infinite slip systems and spherical voids), there is, to this day, no specific guarantee that this estimate will be sufficiently accurate for any void shape and orientation. Nonetheless, we will show in the results sections that the robust character of the original variational method together with the proposed correction in this section gives sufficiently good quantitative agreement when compared with full field finite element simulations for a large range of void shapes and orientations.

\subsection{Summary of the MVAR model and the rate-independent limit}

To facilitate the reader with the main results of the present work, we summarize here the final equations of the proposed MVAR model. Therefore, the effective stress potential of a porous single crystal is given by (see equation (36))

$$
\widetilde{U}_{m v a r}\left(\overline{\boldsymbol{\sigma}} ; s_{\alpha}\right)=(1-f)^{-n} \sum_{s=1}^{K} \frac{\dot{\gamma}_{0}^{(s)}\left(\tau_{0}^{(s)}\right)^{-n}}{n+1}\left(\overline{\boldsymbol{\sigma}} \cdot \widehat{\mathbf{S}}^{\text {mvar },(s)} \cdot \overline{\boldsymbol{\sigma}}\right)^{(n+1) / 2},
$$

where

$$
\widehat{\mathbf{S}}^{\text {mvar },(s)}=\widehat{\mathbf{S}}^{\text {var },(s)}+\left(q_{J}^{2}-1\right) \mathbf{J} \cdot \widehat{\mathbf{S}}^{\text {var },(s)} \cdot \mathbf{J}, \quad q_{J}=\sqrt{\frac{15}{f}}\left\{\frac{(1-f)\left(\beta_{n}\right)^{\frac{1}{n}}}{n\left(f^{-1 / n}-1\right)}\right\}^{\frac{n}{n+1}}, \beta_{n}=\frac{4}{25} 6^{-\frac{n}{2}}
$$

Here,

$$
\widehat{\mathbf{S}}^{\text {var },(s)}=\frac{1}{2} \mathbf{E}^{(s)}+\frac{f}{K} \widehat{\mathbf{S}}^{*}, \quad \mathbf{E}^{(s)}=2 \boldsymbol{\mu}^{(s)} \otimes \boldsymbol{\mu}^{(s)}, \forall s=1, K, \quad J_{i j k l}=\frac{1}{3} \delta_{i j} \delta_{k l} .
$$

where $\boldsymbol{\mu}^{(s)}$ is the Schmid tensor defined in (5) and $\widehat{\mathbf{S}}^{*}$ is evaluated by equations (31)-(34).

In the special case of rate-independent porous single crystals, i.e. $n \longrightarrow \infty$, the MVAR yield criterion is defined via the function $\widetilde{\Phi}_{\text {mvar }}$ as

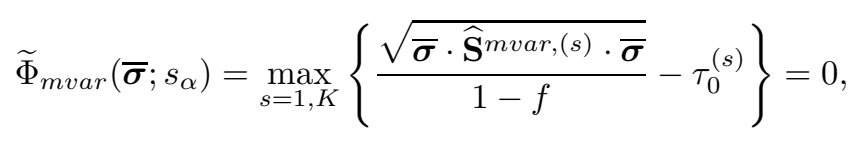

where $\widehat{\mathbf{S}}^{\text {mvar, (s) }}$ is given by (41) with

$$
q_{J}=\sqrt{\frac{5}{2}} \frac{1-f}{\sqrt{f} \ln (1 / f)} .
$$

At this point, it is relevant to mention that the proposed model exhibits many similarities with the 
corresponding MVAR model for porous materials with a $J_{2}$ matrix phase (Aravas and Ponte Castañeda, 2004; Danas and Aravas, 2012) and thus its numerical implementation is expected to be rather straightforward. For this, evolution equations for the microstructural variables need to be provided which is work underway.

\section{Numerical homogenization}

Numerical techniques such as the finite element method are able to solve for the local field in a porous material, provided that the exact location and distribution of the pores is known. However, in most cases of interest, the only available information is the void volume fraction (or porosity) and, possibly, the two-point distribution function of the voids (i.e., isotropic, orthotropic etc). In addition, for sufficient accuracy the element size that should be used in a finite element program must be much smaller than the size of the voids, which in turn is smaller than the size of the periodic unit-cell, especially when multiple pores are considered. Thus, the computation is very intensive in time. Due to all these reasons, it is very difficult to use the numerical results in a multi-scale analysis, especially when the unit-cell is rather complex.

Nevertheless, one could use the numerical periodic homogenization technique as a rigorous test-bed to assess the simpler analytical models as the one proposed in the previous section. More precisely, we can analyze the problem of a periodic porous material considering a unit-cell that contains a given distribution of voids. In this regard, it is well known that a random porous material (e.g., the one in the analytical model presented in the previous section) and the periodic material exhibit similar effective behavior either in the case where the distribution of voids is complex enough (adequate for large porosity) or in the limiting case where the porosity is small enough. Moreover, in these cases, the periodic unitcell estimates, and consequently the effective properties of the periodic composite, are independent of the prescribed periodic boundary conditions (see for instance Gilormini and Michel (1998)). In this regard then, the comparison between the proposed model and the FE periodic unit-cell calculations are meaningful either for complex periodic geometries at finite porosities or for simpler periodic geometries and small to dilute porosities.

The following FE calculations have been carried out with the commercial code Abaqus (Abaqus (2009)) by use of the crystal plasticity user-material subroutine of (Huang (1991); Kysar (1997)).

\subsection{Unit-cell geometries and periodic boundary conditions}

In order to validate the model, as explained before, FE periodic unit-cell calculations need to be carried out. Hence, several unit-cell geometries used in our computations, subjected to periodic boundary conditions, would be presented in this subsection. The present FE calculations are carried out using a small strain formulation since the scope of the study is the estimation of the effective response of the porous crystal with a given microstructural realization but not the evolution of microstructure which is left for a subsequent work. Furthermore, it is important to notice that the entire unit-cell is considered here because of the complex geometry used as well as due to the fact that there exist no planes of symmetry in general, due to the coupling between the crystal anisotropy and the morphological anisotropy.

\subsubsection{Unit-cell geometry}

Before we present the various geometries of the unit cells considered, it is important to note that Fritzen et al. (2012) have shown in the context of porous materials with isotropic matrix that at small porosities $(f \leq 1 \%)$, a simple unit-cell with a single void in the middle gives sufficiently accurate results when compared with those obtained with a unit-cell containing multiple voids randomly distributed. This observation has been verified in the present context of porous single crystals and has been found to hold too. Therefore, no further discussion will be done about this point in the rest of this work. Nonetheless, since the numerical results considered subsequently include porosities of $f=5 \%$ - where a more complex unit cell is necessary - we present briefly below the procedure of creating unit-cells with multiple voids randomly distributed.

More specifically, for spherical voids, we consider a uniform distribution of voids, i.e. multi-void geometries. In this regard, we make use of monodisperse distributions (e.g. fig 2a,b) that are constructed by means of a random sequential adsorption algorithm (see Rintoul and Torquato (1997); Torquato (2002)) which generates the coordinates of the pore centers. Polydisperse distributions of voids 
(Lopez-Pamies et al., 2013) are not necessary at such low porosities. For monodisperse distributions, the radius of each void is

$$
R_{m}=L\left(\frac{3 f}{4 \pi N}\right)^{1 / 3}
$$

with $N$ being the number of pores in the unit-cell and $f$ the porosity.

In addition, the sequential addition of voids is constrained so that the distance between a given void and the rest of the voids as well as the boundaries of the unit-cell takes a minimum value that guaranties adequate spatial discretization. In order to achieve this we have used the rules described in details in (Segurado and Llorca, 2002; Fritzen et al., 2012; Jimenez and Pellegrino, 2012; Lopez-Pamies et al., 2013; López Jiménez, 2014)) and for sake of brevity are not repeated here.

a)

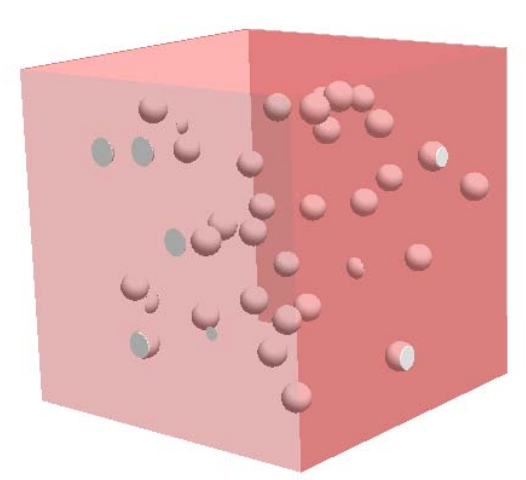

c)

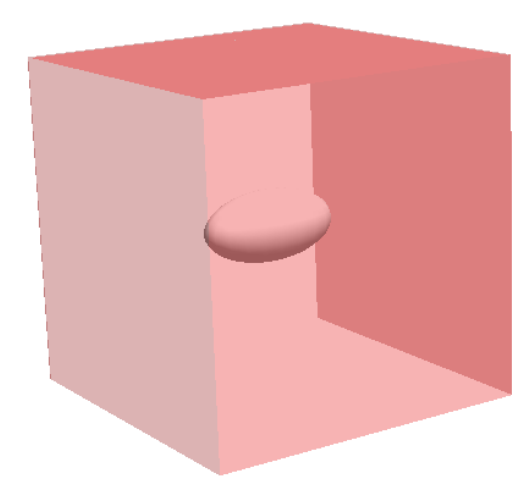

b)

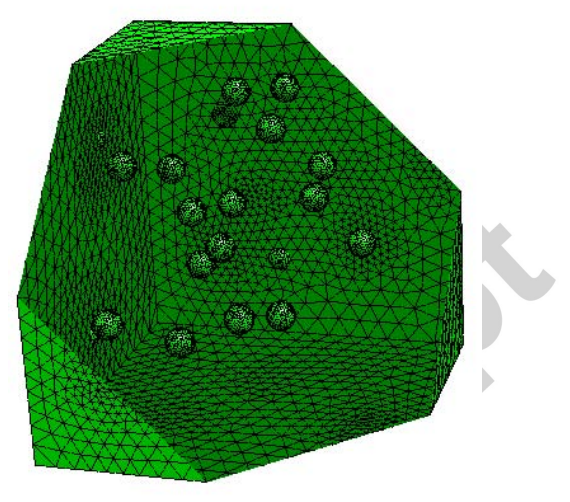

d)

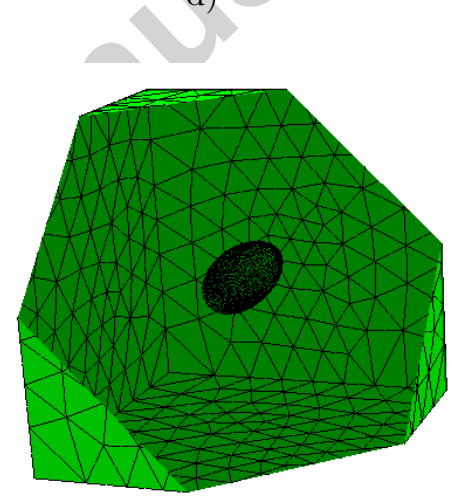

Figure 2: Undeformed unit-cell "square" geometry in the case of (a), (b) a distribution of several spherical voids (c), (d) a single ellipsoidal void.

In the case of ellipsoidal voids, we consider only porosities of $f=1 \%$, and thus geometries with one single void in the middle of the unit-cell are sufficient to estimate the effective behavior of the porous material (see fig 2c,d).

Moreover, it is useful to note that the meshes are made of quadratic ten-node tetrahedral elements $C 3 D 10$ (see Fig. 2b,d), generated with the open source meshing code NETGEN. In the single-void unitcells, a mesh convergence study considered three different meshes, i.e, d.o.f. equal to $10^{4}, 3 \cdot 10^{4}$ and $10^{5}$. The mesh with $\sim 3 \cdot 10^{4}$ d.o.f gave converged results for the average stresses in the unit-cell. In turn, for the multiple void case we considered meshes with $5 \cdot 10^{4}, 10^{5}$ and $5 \cdot 10^{5}$. The one with $10^{5}$ exhibited converged average stresses. Furthermore, in the context of $f=5 \%$, a convergence study with respect to the number of voids per unit cell has also been carried out. By using several unit cells with a number of voids equal to 10,30,50 and 100, we found that convergence of the macroscopic average stress is obtained for 50 voids. 


\subsubsection{Periodic boundary conditions}

In this section, we discuss the application of periodic boundary conditions necessary for the analysis of the above described periodic unit cells. The periodic boundary conditions are expressed in this case as (Michel et al. (1999); Miehe et al. (1999))

$$
\mathbf{v}(\mathbf{x})=\bar{D} \cdot \mathbf{x}+\mathbf{v}^{*}(\mathbf{x}), \mathbf{v}^{*} \text { periodic, }
$$

where the second-order tensor $\overline{\boldsymbol{D}}$ denotes the symmetric part of the average velocity gradient, $\mathrm{x}$ denotes the spatial coordinates and $\mathbf{v}^{*}$ is a periodic field.

Next, one needs to fix one node to cancel the rigid body motion in the FE calculations. For convenience, we choose this node to be at the origin such that $v_{i}(0,0,0)=0(i=1,3)$.

Subsequently, one can subtract the nodal velocities of opposite boundary sides (where $\mathbf{v}^{*}$ is equal, see Aravas (1992)) so that we get the following nodal constraints for the corner nodes of the cube, i.e.,

$$
\begin{aligned}
& v_{i}\left(L_{1}, 0,0\right)-v_{i}(0,0,0)=\bar{D}_{i 1} L_{1}=v_{i}\left(L_{1}, 0,0\right) \\
& v_{i}\left(0, L_{2}, 0\right)-v_{i}(0,0,0)=\bar{D}_{i 2} L_{2}=v_{i}\left(0, L_{2}, 0\right) \\
& v_{i}\left(0,0, L_{3}\right)-v_{i}(0,0,0)=\bar{D}_{i 3} L_{3}=v_{i}\left(0,0, L_{3}\right), \quad \forall i=1,3
\end{aligned}
$$

The above simple relations show that the velocity components of the coner nodes $\left(L_{1}, 0,0\right),\left(0, L_{2}, 0\right)$ and $\left(0,0, L_{3}\right)$ are one-to-one connected to the symmetric part of the average velocity gradient $\bar{D}$. Then, one can write the constraint equations for the rest of the nodes lying on opposite faces of the cube making use of the result (47), i.e.,

$$
\begin{aligned}
& v_{i}\left(L_{1}, x_{2}, x_{3}\right)-v_{i}\left(0, x_{2}, x_{3}\right)=\bar{D}_{i 1} L_{1} \\
& v_{i}\left(x_{1}, L_{2}, x_{3}\right)-v_{i}\left(x_{1}, 0, x_{3}\right)=\bar{D}_{i 2} L_{2} \\
& v_{i}\left(x_{1}, x_{2}, L_{3}\right)-v_{i}\left(x_{1}, x_{2}, 0\right)=\bar{D}_{i 3} L_{3}, \quad \forall i=1,3 .
\end{aligned}
$$

The above algebraic analysis reveals that all periodic linear constraints between all nodes can be written in terms of the velocities of three corner nodes, i.e., $v_{i}\left(L_{1}, 0,0\right), v_{i}\left(0, L_{2}, 0\right)$ and $v_{i}\left(0,0, L_{3}\right)$, which, in turn, are given in terms of $\overline{\boldsymbol{D}}$ by equation (47). This, further, implies that the only nodes that boundary conditions need to be applied are lying on opposite faces of the cube $\left(L_{1}, 0,0\right),\left(0, L_{2}, 0\right)$ and $\left(0,0, L_{3}\right)$ (together with the axes origin $(0,0,0)$ which is fixed).

\subsection{Loading through stress triaxiality control}

In order to validate the model proposed in this study, it is convenient to apply $\bar{D}$ in such a way that certain stress components in the unit-cell remains constant.

In particular, we discuss the methodology for the application of a constant average stress triaxiality and constant average Lode angle in the unit-cell. This methodology has been originally proposed by (Barsoum and Faleskog (2007); Dunand and Mohr (2014)) and further discussed in (Mbiakop et al. (2015a)). While general loading conditions can be applied in the periodic unit-cells described above we will only restrict attention here to purely triaxial loads and general crystal anisotropies and void shapes. On the other hand, the principal components of the stress field can be expressed as a function of the average stress triaxiality $X_{\Sigma}=\bar{\sigma}_{m} / \bar{\sigma}_{e q}$ and the average Lode angle $\theta$, via

$$
\frac{3}{2 \bar{\sigma}_{e q}}\left\{\bar{\sigma}_{1}, \bar{\sigma}_{2}, \bar{\sigma}_{3}\right\}=\left\{\cos \bar{\theta},-\cos \left(\bar{\theta}+\frac{\pi}{3}\right),-\cos \left(\bar{\theta}-\frac{\pi}{3}\right)\right\}+\frac{3}{2} X_{\Sigma}\{1,1,1\} .
$$

where $\bar{\sigma}_{e q}$ denotes the equivalent Von Mises part of $\overline{\boldsymbol{\sigma}}$.

As a consequence of above-defined load and the periodic boundary conditions, the average deformation in the unit-cell is entirely described by the displacements of the three corner nodes, e.g., $u_{1}\left(L_{1}, 0,0\right)=$ $U_{1}(t), u_{2}\left(0, L_{2}, 0\right)=U_{2}(t)$ and $u_{3}\left(0,0, L_{2}\right)=U_{3}(t)$, denoted compactly as

$$
\mathbf{U}=\left\{U_{1}(t), U_{2}(t), U_{3}(t)\right\}, \quad \dot{\mathbf{U}}=\left\{\dot{U}_{1}(t), \dot{U}_{2}(t), \dot{U}_{3}(t)\right\} \equiv\left\{v_{1}(t), v_{2}(t), v_{3}(t)\right\} .
$$

Recalling that the average strain-rate and stress tensors involve only two non-zero components due to 
the applied triaxial loading, they can be expressed in vectorial form (i.e., using the Voigt notation) as

$$
\overline{\boldsymbol{D}}=\left\{\frac{\dot{U}_{1}}{L_{1}+U_{1}}, \frac{\dot{U}_{2}}{L_{2}+U_{2}}, \frac{\dot{U}_{3}}{L_{3}+U_{3}}\right\}, \quad \overline{\boldsymbol{\sigma}}=\left\{\bar{\sigma}_{1}, \bar{\sigma}_{2}, \bar{\sigma}_{3}\right\} .
$$

To proceed further, we rewrite the average strain-rate tensor as

$$
\overline{\boldsymbol{D}}=\mathcal{Q}^{-1} \cdot \dot{\mathbf{U}}, \quad \mathcal{Q}=\operatorname{diag}\left(L_{1}+U_{1}, L_{2}+U_{2}, L_{3}+U_{3}\right),
$$

where $\mathcal{Q}$ is diagonal matrix of dimension three.

We, next, define an external fictitious node ${ }^{3}$, whose generalized force, $\mathbf{P}_{G}$, and generalized displacement, $\mathbf{p}_{G}$, vectors, respectively, take the form

$$
\mathbf{P}_{G}=\left\{P_{1}^{G}(t), 0,0\right\}, \quad \mathbf{p}_{G}=\left\{p_{1}^{G}(t), p_{2}^{G}(t), p_{3}^{G}(t)\right\} .
$$

The stress state in the unit-cell is then controlled via a time-dependent kinematic constraint (Michel et al., 1999) obtained by equilibrating the rate of work in the unit-cell with the rate of work done by the fictitious node on the unit-cell at time $t$, such that

$$
\dot{W}=V \overline{\boldsymbol{\sigma}} \cdot \boldsymbol{D}=\mathbf{P}_{G} \cdot \dot{\mathbf{p}}_{G} .
$$

Next, in order to control the loading path in the stress space, we couple the average stress $\boldsymbol{\sigma}$ in the unitcell with the generalized force vector associated with the fictitious node $\mathbf{P}_{G}$ via the constraint equation

$$
V \overline{\boldsymbol{\sigma}}=\mathbf{C} \cdot \mathbf{P}_{G}, \quad \mathbf{C}=\left(\frac{\mathbf{c}_{1}}{\left|\mathbf{c}_{1}\right|} ; \frac{\mathbf{c}_{2}}{\left|\mathbf{c}_{2}\right|} ; \frac{\mathbf{c}_{3}}{\left|\mathbf{c}_{3}\right|}\right)^{T}, \quad \mathbf{C}^{-1}=\mathbf{C}^{T},
$$

where $\mathbf{C}$ is a non-dimensional proper orthogonal matrix since $\mathbf{c}_{i}(\mathrm{i}=1,2,3)$ are three dimensional vectors that form an orthogonal basis set. The vectors $\mathbf{c}_{i}(\mathrm{i}=1,2,3)$ depend on the three components of the average stress $\sigma$, such that

$$
\mathbf{c}_{1}=\left\{\bar{\sigma}_{1}, \bar{\sigma}_{2}, \bar{\sigma}_{3}\right\}, \quad \mathbf{c}_{2}=\left\{\bar{\sigma}_{1} \bar{\sigma}_{3}, \bar{\sigma}_{2} \bar{\sigma}_{3},-\left(\bar{\sigma}_{1}^{2}+\bar{\sigma}_{2}^{2}\right)\right\}, \quad \mathbf{c}_{3}=\left\{-\bar{\sigma}_{2}, \bar{\sigma}_{1}, 0\right\},
$$

with $\bar{\sigma}_{i}, i=1,2,3$ given in equation (49).

The above expressions for the vectors $\mathbf{c}_{i}(\mathrm{i}=1,2,3)$ further imply that the matrix $\mathbf{C}$ in equation (55) is only a function of the stress triaxiality $X_{\Sigma}$ and the Lode angle $\theta$ but not of the equivalent stress $\bar{\sigma}_{e q}$. By substitution of equations (52) and (55) in (54), one gets

$$
\dot{\mathbf{U}}=\mathcal{Q} \cdot \mathbf{C} \cdot \dot{\mathbf{p}}_{G} .
$$

The above expression provides the kinematic constraints between the degrees of freedom corresponding to the sides of the unit-cell (i.e., $\mathbf{U}$ ) and the degrees of freedom of the fictitious node (i.e., $\mathbf{p}_{G}$ ). The calculation is done then by prescribing the $X_{\Sigma}, \bar{\theta}$ and a loading history of $\dot{\mathbf{p}}_{G}$. These nonlinear constraints are applied in the finite element software ABAQUS (Abaqus, 2009) by use of the multi-point constraint user subroutine (MPC).

\section{Results - I: Assessment of the MVAR model via FE simulations}

This section presents results for the effective behavior of rate-dependent porous single crystals as predicted by the modified variational model (MVAR) proposed in this work. The predictions of the MVAR are compared with corresponding results obtained by the FE simulations described in section 4 . Before proceeding with the discussion of the results, it is useful to introduce first the various material and loading parameters used in the following figures. The present study investigates a range of creep exponents $n=(1,2,5,10)$, porosities $f=(1 \%, 5 \%)$, void shapes and orientations (e.g., spherical and ellipsoidal) as well as Face-Centered Cubic (FCC), Body-Centered Cubic (BCC) and Hexagonal Closed Packed (HCP) single crystals. These choices of single crystals correspond to the large majority of metals.

\footnotetext{
${ }^{3}$ The fictitious node introduced in the present study has no specific physical interpretation, but serves only as a mathematical tool to apply the required boundary conditions at the unit-cell.
} 
In addition, motivated by common practice in crystal plasticity studies, we consider the case where all the slip systems of the matrix have the same critical resolved shear stress (CRSS) and reference slip-rate, as defined in equation (12). The crystallographic orientation will be defined independently in the following sections.

In turn, we will focus on two different types of loading conditions. In the first one, the principal directions of the macroscopic stress tensor $\overline{\boldsymbol{\sigma}}$, or equivalently $\overline{\boldsymbol{\Sigma}}$ are aligned with the fixed Cartesian laboratory frame of reference defined by the three unit vectors $\mathbf{e}^{(i)}, i=1,2,3$. In this case, one could rewrite the principal stresses in terms of the average equivalent von Mises stress, $\bar{\Sigma}_{e q}$, the average (macroscopic) Lode angle, $\bar{\theta}$, and the average stress triaxiality $X_{\Sigma}$. This reads, in diagonal matrix notation

$$
\bar{\Sigma}=\bar{\Sigma}_{m}\{1,1,1\}+\frac{2}{3} \bar{\Sigma}_{e q}\left\{\cos \bar{\theta},-\cos \left(\bar{\theta}+\frac{\pi}{3}\right),-\cos \left(\bar{\theta}-\frac{\pi}{3}\right)\right\} .
$$

The second set of loading conditions involves the two average shear components $\bar{\Sigma}_{13}$ and $\bar{\Sigma}_{23}$ with superimposed hydrostatic stressing $\bar{\Sigma}_{m}$, such that (in tensorial notation)

$$
\bar{\Sigma}=\bar{\Sigma}_{m} \sum_{i=1}^{3} \mathbf{e}^{(i)} \otimes \mathbf{e}^{(i)}+\bar{\Sigma}_{13}\left(\mathbf{e}^{(1)} \otimes \mathbf{e}^{(3)}+\mathbf{e}^{(3)} \otimes \mathbf{e}^{(1)}\right)+\bar{\Sigma}_{23}\left(\mathbf{e}^{(2)} \otimes \mathbf{e}^{(3)}+\mathbf{e}^{(3)} \otimes \mathbf{e}^{(2)}\right) .
$$

Since the response of the porous single crystal is anisotropic these are simply two out of several possible sets of loading conditions. Nevertheless, as we will show in the following, they are sufficient to reveal the most important features of the porous single crystal response.

Furthermore, it is important to clarify that the porous crystal exhibits two types of anisotropy. The first is that of the crystal matrix as a result of the corresponding slip systems and the second is that of the void shape and orientation, which is ellipsoidal in general. Therefore, the effective response of the porous crystal is a function of all the six stress components. Of course showing cross-sections with all possible combination of stress components is to cumbersome and of a little value to the understanding of the porous single crystal response. Thus, following traditional notation in the context of porous materials, as well as similar studies of Han et al. (2013); Paux et al. (2015), the following results are broken down into three major groups following the above mentioned loading conditions. The first group shows the effective response of the porous crystals in the space $\bar{\Sigma}_{m}-\bar{\Sigma}_{e q}$. This stress space is very important since it involves directly the dependence upon the hydrostatic component $\bar{\Sigma}_{m}$. The second group shows results in deviatoric planes such as for instance the one defined by the out-of-plane shear stresses $\bar{\Sigma}_{13}$ $\bar{\Sigma}_{23}$, for $\bar{\Sigma}_{12}=0$ and for given constant hydrostatic stress $\bar{\Sigma}_{m}$. This cross-section reveals the non trivial coupling of crystal anisotropy and void shape (morphological) anisotropy. The last group displays results in the П-plane (or octahedral plane), defined by polar coordinates $(r, \phi)=\left(\bar{\Sigma}_{e q}, \bar{\theta}\right)$ at different levels of superimposed pressure $\bar{\Sigma}_{m}$. These choices lead to more complete results in the sense that they cover a significant range of the stress space but at the same time using traditional and well-understood stress measures.

\subsection{Computation of the gauge surface in the numerical homogenization}

The evaluation of the gauge surfaces resulting from the numerical computations can be expressed using the definition (18) but its evaluation is non trivial and is described in the following. The general idea follows from the earlier study of Flandi and Leblond (2005) but is rather different since it has to be appropriately modified to apply for the general commercial code Abaqus used in the present study.

By making use of the homogeneity of degree $n+1$ in $\bar{\Sigma}$ of the function $\widetilde{U}$ (see (2)), one can write

$$
\widetilde{U}\left(\bar{\Sigma} ; s_{\alpha}\right)=\left(\bar{\Sigma}_{e q}\right)^{n+1} \widetilde{U}_{N}\left(\boldsymbol{N}, X_{\Sigma} ; s_{\alpha}\right)=\frac{\dot{\gamma}_{0} \tau_{0}^{-n}}{n+1},
$$

while $\boldsymbol{N}=\overline{\boldsymbol{\Sigma}}^{d} / \bar{\Sigma}_{e q}$ describes the loading direction in the deviatoric space, with $\overline{\boldsymbol{\Sigma}}^{d}$ and $\bar{\Sigma}_{e q}$ denoting the deviatoric and equivalent Von Mises part of the average stress $\bar{\Sigma}$, respectively, corresponding to the equi-potential surface (18).

Next, given any average stress, $\overline{\boldsymbol{\sigma}}$, one can write

$$
\widetilde{U}\left(\overline{\boldsymbol{\sigma}} ; s_{\alpha}\right)=(1-f) \min _{\boldsymbol{\sigma} \in S(\overline{\boldsymbol{\sigma}})} \frac{1}{V\left(\Omega^{(1)}\right)} \int_{\Omega^{(1)}} U(\boldsymbol{\sigma}) d V=\left(\bar{\sigma}_{e q}\right)^{n+1} \widetilde{U}_{N}\left(\boldsymbol{N}, X_{\Sigma} ; s_{\alpha}\right) .
$$


Due to the homogeneity of $\widetilde{U}$, it is noted that $\widetilde{U}_{N}\left(\boldsymbol{N}, X_{\Sigma} ; s_{\alpha}\right)$ is the same function in (60) and (61), and hence combination of these two equations gives

$$
\bar{\Sigma}_{e q}=\left[(n+1) \dot{\gamma}_{0}^{-1} \tau_{0}^{n} \widetilde{U}\left(\overline{\boldsymbol{\sigma}} ; s_{\alpha}\right)\right]^{\frac{-1}{n+1}} \bar{\sigma}_{e q}
$$

In the FE code, $\bar{D}_{11}$ and the average $X_{\Sigma}$ in the unit-cell are applied and remain constant throughout the process, as discussed in section 4.2 , whereby $\bar{D}_{22}, \bar{D}_{33}$ and $\bar{\sigma}_{e q}$ are calculated. In addition, postprocessing of the numerical results provides $\widetilde{U}\left(\overline{\boldsymbol{\sigma}} ; s_{\alpha}\right.$ ) (via definition (2)). Finally, $\bar{\Sigma}_{e q}$ is readily obtained from equation (62), while $\bar{\Sigma}_{m}=X_{\Sigma} \bar{\Sigma}_{e q}$ given the prescribed average stress triaxiality $X_{\Sigma}$ in the unit-cell.

\subsection{Porous crystals with spherical voids}

\subsubsection{Face-Centered Cubic single crystal}

In this section Face-Centered Cubic (FCC) single crystals are considered. In the FCC crystalline structure, plastic slip occurs on a group of 12 slip systems following $\{111\}\langle 110\rangle$ (see Table 1).

\begin{tabular}{|c|c|c|c|c|c|c|c|c|c|c|c|c|}
\hline slip system $\mathrm{s}$ & 1 & 2 & 3 & 4 & 5 & 6 & 7 & 8 & 9 & 10 & 11 & 12 \\
\hline slip plane $\mathbf{~ m}$ & & $(111)$ & & & $(111)$ & & & $(\overline{111)}$ & & & $(11 \overline{1})$ & \\
\hline slip direction $\mathbf{s}$ & [101] & {$[0 \overline{1} 1]$} & [110] & {$[\overline{1} 01]$} & [011] & {$[110]$} & {$[0 \overline{1} 1]$} & [110] & {$[101]$} & [110] & {$[101]$} & [011] \\
\hline
\end{tabular}

Table 1: Slip systems in FCC crystalline structure.

In this loading configuration, Fig. 3 shows cross-sections of the effective gauge surfaces in the $\bar{\Sigma}_{m}-\bar{\Sigma}_{e q}$ plane, for a creep exponent $n=10$, a porosity $f=1 \%$ and several Lode angles $\bar{\theta}=0^{\circ}, 10^{\circ}, 30^{\circ}$. As we can observe, the MVAR gauge surfaces are symmetric with respect to the $\bar{\Sigma}_{e q}$ axis for all the Lode angles considered. On the other hand, the FE computations predict symmetric gauge surfaces for $\bar{\theta}=0^{\circ}, 30^{\circ}$ (see Fig. 3a,c) whereas a rather small "asymmetry" with respect to the $\bar{\Sigma}_{e q}$ axis is observed for $\bar{\theta}=10^{\circ}$ (see Fig. 3b). Following the observations of this figure for spherical voids, which indicate only a minor asymmetry of the gauge surface and only in some cases, we will choose in most of the results presented in the sequel a Lode angle $\bar{\theta}=0^{\circ}$. Therefore, for this case of spherical voids only one quarter of the surface is shown.

a)

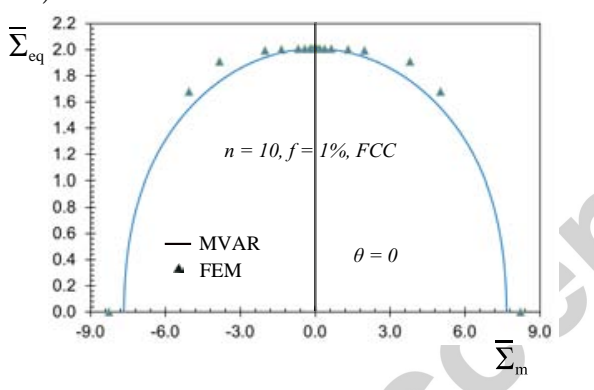

b)

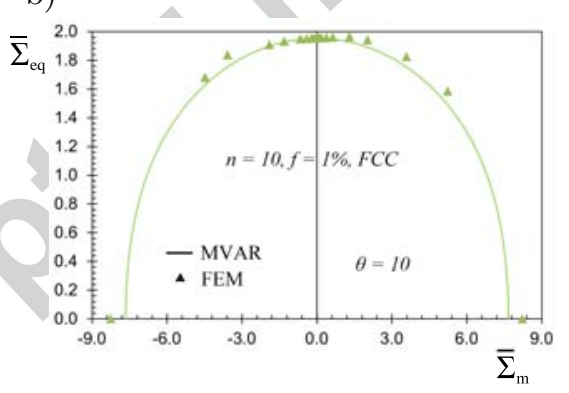

c)

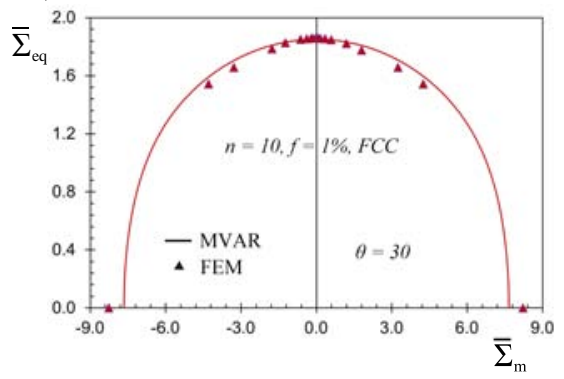

Figure 3: Gauge surfaces in the $\bar{\Sigma}_{m}-\bar{\Sigma}_{e q}$ plane in the case of a porous FCC single crystal comprising spherical voids, for a creep exponent $n=10$ and a porosity $f=1 \%$. Comparison between the model (MVAR) and the FE results for Lode angles $\bar{\theta}=0^{\circ}, 10^{\circ}, 30^{\circ}$.

Specifically, Figure 4 shows cross-sections of the effective gauge surfaces in the $\bar{\Sigma}_{m}-\bar{\Sigma}_{e q}$ plane. Various creep exponents $n=(1,2,5,10)$ are considered while the porosity is set to $f=1 \%$ (Fig. 4a) and $f=5 \%$ (Fig. $4 \mathrm{~b}$ ). In the context of this figure, we observe a very good agreement between the MVAR predictions and the FE results for the entire range of creep exponents $n$. More precisely, the agreement between the MVAR predictions and the FE results is very good for small nonlinearities $n=1,2$, whereas it slightly tends to underestimate the effective response at higher ones (i.e., $n=5,10$ ) and for very large triaxialities. In any case, the maximum error is found to be in the order of $\sim 5 \%$. For $f=1 \%$, in Fig. $4 \mathrm{a}$, and relatively high triaxialities, the $n=1$ curve crosses the rest of the curves leading to a stiffer response at large stress triaxialities $X_{\Sigma}$. This feature is validated qualitatively by the FE computations, but quantitatively may involve errors from both the FE calculations as well as inaccuracies of the MVAR model in this regime. 


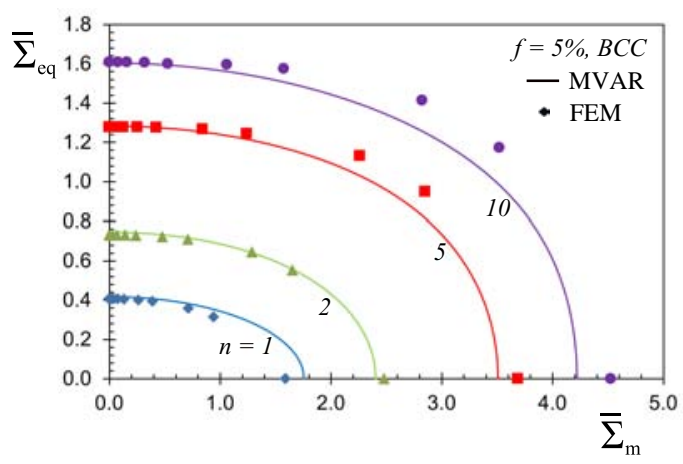

Figure 5: Gauge surfaces in the $\bar{\Sigma}_{m}-\bar{\Sigma}_{e q}$ plane in the case of a porous BCC single crystal comprising spherical voids, for a Lode angle $\bar{\theta}=0$ and a range of creep exponent $n=(1,2,5,10)$. Comparison between the model (MVAR) and the FE results for a porosity (a) $f=1 \%$, (b) $f=5 \%$. 


\subsubsection{Hexagonal Closed Packed single crystal}

In the context of HCP porous crystals, we consider three distinct cases as described in Table 3 . Those three cases are chosen such that the crystal goes from an extremely anisotropic response (case $C_{1}$ in Table 3) to those that involve a larger number of active slip systems, i.e., cases $C_{2}$ and $C_{3}$ in Table 3.

It is noted here that for the cases $C_{2}$ and $C_{3}$, we consider the loading described in equation (58), whereby for the case $C_{1}$ we apply the loading defined in equation (59). The reason is that for the $C_{1}$ porous crystal application of the triaxial load (58) leads to no overall macroscopic plasticity (i.e., the average stress measures do not saturate). While one could claim that such a low number of slip systems (e.g., three basal) are not sufficient to accommodate a general loading state in a uniform material, it is not the case in a composite material. In other words, due to the presence of the vacuous phase, the stress state near the void surface is complex enough (gives rise to nonzero shear stresses) to activate the available slip systems locally for any choice of the macroscopic loading. Even so, the resulting average stress in the unit-cell does not saturate implying the the homogenized response of the unit cell does not lead to effective plasticity. The proposed homogenization model has this property as we will see in the sequel.

Let us consider first the case $C_{1}$ in Table 3 where plastic slip occurs only in the so called basal planes, such as for instance pure Titanium. The crystalline structure is consequently $\{0001\}\langle 1 \overline{2} 10\rangle$. In this case, rather surprisingly, the computation of the microstructural tensors in equation (31) leads to $\widehat{\mathbf{S}}^{*}=\mathbf{0}$, and thus, the MVAR estimate (40) becomes

$$
\widetilde{U}_{\text {mvar }}\left(\overline{\boldsymbol{\sigma}} ; s_{\alpha}\right)=(1-f)^{-n} \sum_{s=1}^{K} \frac{\dot{\gamma}_{0}^{(s)} \tau_{0}^{(s)}}{n+1}\left(\frac{\left|\overline{\boldsymbol{\sigma}} \cdot \boldsymbol{\mu}^{(s)}\right|}{\tau_{0}^{(s)}}\right)^{n+1}
$$

This implies that the MVAR estimate leads to a fully incompressible response in this case $C_{1}$, irrespective

\begin{tabular}{|c|c|c|c|c|}
\hline Cases & Macro-loading & slip plane $\mathbf{m}$ & slip direction s & number of slip systems \\
\hline$C_{1}$ & Eq.(59) & Basal $\{0001\}$ & Type $\langle a\rangle\langle 1210\rangle$ & 3 \\
\hline$C_{2}$ & Eq.(58) & Pyramidal $\Pi_{2}\{11 \overline{2} 2\}$ & Type $\langle c+a\rangle\langle 11 \overline{2} 3\rangle$ & 6 \\
\hline \multirow{2}{*}{$C_{3}$} & Eq. $(58)$ & Basal $\{0001\} \&$ & Type $\langle a\rangle\langle 1210\rangle \&$ & \\
& & $\begin{array}{c}\text { Prismatic }\{10 \overline{1} 0\} \& \\
\text { Pype }\langle a\rangle\langle 1 \overline{2} 10\rangle \&\end{array}$ & $3+3+6=12$ \\
& & Type $\langle c+a\rangle\langle 11 \overline{2} 3\rangle$ & \\
\hline
\end{tabular}

Table 3: Slip systems in some hexagonal crystalline structures.

of the value of porosity, void shape or orientation used, as shown in Fig. 6a. The deviatoric part, on the other hand, is affected by the voids while the resulting stress potential (63) has the exact form of the stress potential of the matrix (10) weakened only by a factor that depends on $f$ and the creep exponent $n$. It is important to mention at this point that even though the crystal matrix has only a few active systems, the porous composite can, in general, accommodate all possible loads since the vacuous phase is fully isotropic and compressible. Thus, the fact that an HCP porous crystal with only three active systems still remains incompressible is a highly non-trivial result. The explanation of such a response can be attributed to virtual "rigid" directions in the composite thus leading to pressure-independent response. To investigate this further, we consider the cases of HCP singles crystal with pyramidal $\Pi_{2}$ $\left(C_{2}\right.$ in Table 3$)$ and combination of basal, prismatic and pyramidal $\Pi_{2}$ active slip systems $\left(C_{3}\right.$ in Table 3$)$, as shown in Table 3. In these cases where more slip systems are activated, the MVAR predictions lead gradually to more compressible responses. In other words, in Fig. 6a, one observes that the HCP crystal $C_{3}$, with 12 slip systems is more compressible than the HCP crystal $C_{2}$, with 6 slip systems. These predictions are fully confirmed via corresponding FE calculations, as shown in Fig. 6a.

In turn, in Fig. $6 \mathrm{~b}$, we show the effect of the creep exponent $n=1,2,5,10$ for an HCP crystal with $K=6$ slip systems that is described by case $C_{2}$ in Table 3 . The agreement between the MVAR predictions and the FE results is relatively good for all exponents $n$ shown here. More interestingly, perhaps, there exists a crossing of the gauge curves as one goes from low to high triaxialities. For instance, the while at low $X_{\Sigma}$ the $n=1$ curve is more compliant than that of $n=10$, the inverse occurs at higher triaxialities. This feature is completely validated qualitatively and quantitatively by the FE computations. Direct comparison between the case of HCP with $K=6$ (Fig. 6b) and those for FCC (Fig. 4a) and BCC (Fig. 5a) reveal the nontrivial coupling between the creep exponent $n$, the presence of the pores and the slip system orientation. 
Figure 6: Gauge surfaces in the $\bar{\Sigma}_{m}-\bar{\Sigma}_{e q}$ plane in the cases of (a) three porous HCP single crystal (with $K=3$, $K=6$ and $K=12$ slip systems) comprising spherical voids, for the loading (59) in case $C_{1}$ and the loading (58) and Lode angle $\bar{\theta}=0$ for the cases $C_{2}$ and $C_{3}$, a porosity $f=1 \%$ and a creep exponent $n=10$ and (b) for different exponents $n=1,2,5,10$ and $K=6$ slip systems subjected to the loading (58) and Lode angle $\bar{\theta}=0$.

To summarize, the previous cases of several HCP crystalline structures show that the MVAR model deals extremely well with the strong sensitivity of the porous single crystals behavior on the crystal anisotropy (i.e., number and orientation of slip systems), as already discussed in Mbiakop et al. (2015b) in the context of two-dimensional microstructures. This implies that by default the present model is able to distinguish with high accuracy between different crystals (and effective loading). Even though this choice of slip systems is somewhat theoretical in nature, the fact that for HCP with 3 slip systems the response is incompressible as well as that 6 slip systems lead to less pressure-dependence than 12 slip systems has strong implications on void growth. Moreover, it is important to mention here that in real HCP crystals the different sets of slip systems (i.e., basal, pyramidal, etc) could exhibit different critical resolved shear stresses, $\tau_{0}^{(s)}$. This case is not studied here since the goal is to present more general qualitative features of the model. However, such effects could be readily considered in the present framework, since the MVAR model is general.

\subsection{Porous crystals with ellipsoidal voids}

In this section, we show results for porous single crystals comprising ellipsoidal voids, i.e., with aspect ratios $w_{1} \neq 1$ and $w_{2} \neq 1$. The microstructure considered here is defined by porosity $f=1 \%$, void aspect ratios $w_{1}=w_{2}=3$ and void orientations $\mathbf{n}^{(1)}=\mathbf{e}^{(1)}, \mathbf{n}^{(2)}=\mathbf{e}^{(2)}, \mathbf{n}^{(3)}=\mathbf{e}^{(3)}$. Figure 7 presents various cross-sections of the effective gauge surfaces in the $\bar{\Sigma}_{m}-\bar{\Sigma}_{e q}$ plane in the case of creep exponents $n=1,10$, for FCC and BCC single crystals, respectively. In the context of this figure, the MVAR predictions are in relatively good agreement with the FE results for both $n=1,10$. In particular, for $n=1$, the MVAR is in excellent agreement with the FE results except at purely hydrostatic loadings where an error in the order of $8 \%$ is observed. Nonetheless, in that case numerical convergence issues appeared and the FE results should be interpreted with caution. In turn, when $n=10$, the MVAR exhibits very good qualitative agreement with the FE results where the corresponding gauge surface exhibits a rather weak "asymmetry" with respect to the $\bar{\Sigma}_{e q}$ axis, while it tends to be more compliant with respect to the numerical estimates at moderate and high triaxialities. This asymmetry, which is present in the case of ellipsoidal voids, is a direct consequence of the coupling between $\bar{\Sigma}_{m}$ and the shear stresses resulting from the complex form of the tensor $\widehat{\mathbf{S}}^{*}$ defined in equation 31 . Such effects observed in shearing of ellipsoidal voids have also been addressed either in numerical micromechanical calculations (see for instance Tvergaard and Nielsen (2010)), or in multiaxial experiments (see Combaz et al. (2011)).

\subsection{Full field contours for periodic unit-cells}

In order to have a better understanding on the differences between gauge surfaces for several crystal anisotropies (FCC, BCC, HCP), we present, next, contours of the maximum principal logarithmic strain, for spherical voids, a creep exponent $n=5$, a porosity $f=1 \%$ and a triaxiality $X_{\Sigma}=3$. As displayed in Fig. 8, the strain amplitude is in most of the unit-cell regions lower in the case $C_{1}$ (see Table 3 ) with $K=3 \mathrm{HCP}$ slip systems than in the FCC single crystal which is itself lower than in the BCC single crystal. These observations can explain at one hand the incompressible macroscopic response of the HCP 
Figure 7: Gauge surfaces in the $\bar{\Sigma}_{m}-\bar{\Sigma}_{e q}$ plane for a porous single crystal comprising ellipsoidal voids of $f=1 \%$, a Lode angle $\bar{\theta}=0$, void aspect ratios $w_{1}=w_{2}=3$, void orientations $\mathbf{n}_{(1)}=\mathbf{e}_{(1)}, \mathbf{n}_{(2)}=\mathbf{e}_{(2)}, \mathbf{n}_{(3)}=\mathbf{e}_{(3)}$ and a range of creep exponent $n=(1,10)$. Comparison between the model (MVAR) and the FE results in the cases of (a) FCC, (b) BCC.

porous single crystal, but on the other hand the fully compressible response of the BCC and FCC single crystals.

Moreover, we observe that pore interaction and deformation localization can becomes rather significant leading to several completely unloaded regions around a significant number of pores. This effect of strong field fluctuations is much less pronounced for higher number of slip systems $K=48$ (BCC) as shown in Fig. 8a. The observed deformation localization also explains why the MVAR model tends to underestimate effective response when compared with the FE results at increasing creep exponent $n$ and increasing porosity $f$. Such effects have already been observed in porous materials with isotropic matrix and high nonlinearity (see for instance Idiart et al. (2006)). In view of this, more advanced models incorporating better description of these field fluctuations (see for instance Danas and Ponte Castañeda (2009a)) could possibly yield a better agreement with a cost of more complexity in the obtained model. In turn, one could calibrate the present MVAR model similar to the work of Cao et al. (2015) retaining the simplicity. Such an attempt is done in Section 7, where it is shown that such calibration can increase the accuracy of the MVAR model at very high nonlinearities such as the rate-independent limit.

a)

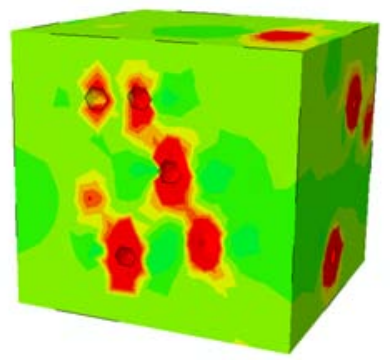

$\mathrm{BCC}$

\section{Maximum principal strain}

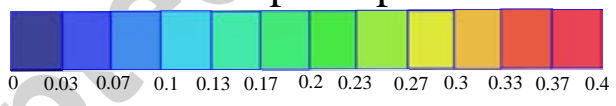

b)

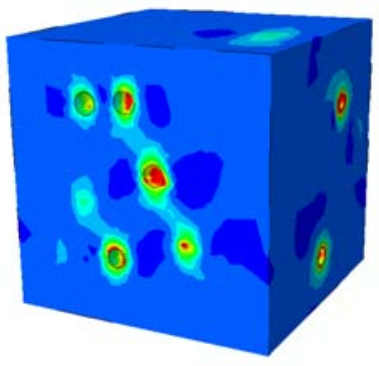

FCC c)

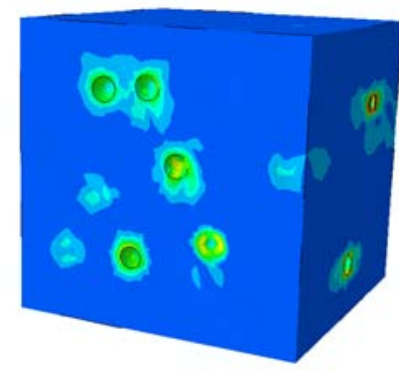

$\mathrm{HCP}, K=3$

Figure 8: Contour of the maximum principal logarithmic strain for a porous single crystal with a "multipore" geometry, a creep exponent $n=5$, a porosity $f=1 \%$ a triaxiality $X_{\Sigma}=3$ and a Lode angle $\bar{\theta}=0$. Case of (a) BCC (b) FCC and (c) HCP, $K=3$. 


\section{Results - II: Coupling between crystal anisotropy, void shape and orientation}

In the previous sections, the MVAR model has been assessed in great detail and it was found to be in good agreement with the corresponding $\mathrm{FE}$ results. In this section, we will focus on MVAR estimates only and explore the effect of a large number of parameters including different loading states, nonlinearities and void orientations. The goal is to reveal the complex coupling between the crystal anisotropy (FCC, BCC, HCP), and the (morphological) void anisotropy resulting from the ellipsoidal void shape and orientation, using the MVAR model without insisting on quantitative aspects.

\subsection{Effect of the crystal anisotropy}

Fig. 9 shows gauge surfaces in the $\bar{\Sigma}_{m}-\bar{\Sigma}_{e q}$ plane of FCC and BCC porous single crystals with spherical voids $\left(w_{1}=w_{2}=1\right)$, a range of porosities $f=(1 \%, 5 \%, 10 \%)$, various creep exponents $n=10$ and $n \rightarrow \infty$ (rate independent case). We observe a significant dependence of the average deviatoric response on the crystal anisotropy (FCC, BCC) either in the rate-dependent $(n=10)$ or in the rateindependent $(n \rightarrow \infty)$ context. Rather interestingly, the hydrostatic point for BCC and FCC coincides in the case of rate-independent context $(n \longrightarrow \infty)$ but such a feature is not preserved in the rate dependent regime (finite $n$ values). This important result is of special interest to limit analysis approaches which are derived in the rate-independent limit and are extended to the rate-dependent limit in a heuristic manner.

a)

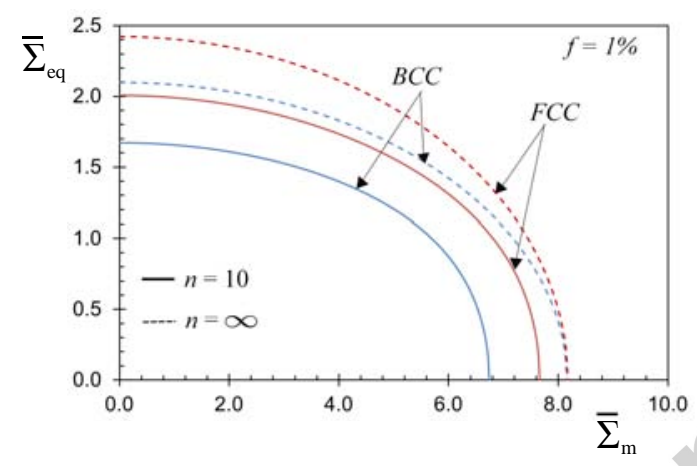

b)

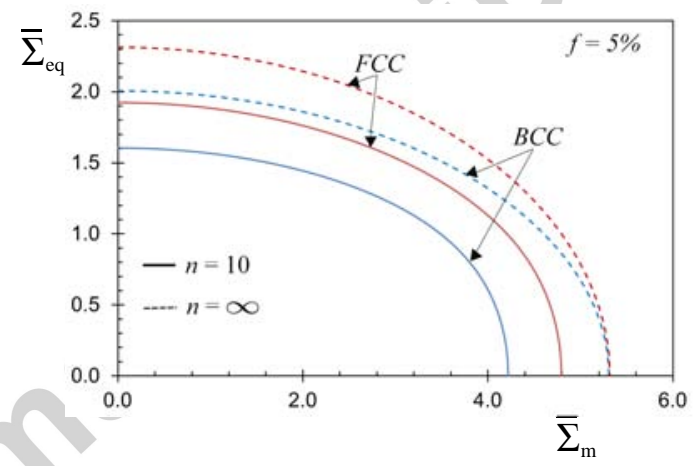

Figure 9: Gauge surfaces in the $\bar{\Sigma}_{m}-\bar{\Sigma}_{e q}$ plane for porous FCC and BCC single crystals with spherical voids, a Lode angle $\bar{\theta}=0$, various creep exponents $n=10$ (continuous lines) and $n \rightarrow \infty$ (dashed lines). Cases of (a) $f=1 \%$, (b) $f=5 \%$.

The effect of matrix crystal anisotropy upon the effective response of the porous composite is discussed next. As already observed in the context of Fig. 6, where spherical voids are embedded in several HCP crystal structures, the average hydrostatic response is strongly influenced by the number and orientation of the slip systems. Specifically, both the MVAR model and the FE results predict that the effective behavior of a $\mathrm{HCP}$ porous single crystal with $K=3$ three basal slip systems (i.e., highly anisotropic case $C_{1}$ in Table 3 ) leads to a completely incompressible response while it exhibits a compressible one for HCP porous crystals with $K=6$ pyramidal $\Pi_{2}$ slip systems (case $C_{2}$ in Table 3 ) as well as with a combination of 3 basal, 3 prismatic and 6 pyramidal $\Pi_{2}$ slip systems (case $C_{3}$ in Table 3 ).

On the other hand, let us considers now, as shown in Fig. 10, gauge surfaces in a deviatoric $\bar{\Sigma}_{13}-\bar{\Sigma}_{23}$ plane of HCP (with $K=3$ slip systems), FCC and BCC porous single crystals with spherical voids ( $w_{1}=$ $w_{2}=1$ ), a porosity $f=5 \%$ and a creep exponent $n=10$. The various cross-sections Fig. 10a, Fig. 10b and Fig. 10c correspond to different hydrostatic stresses $\bar{\Sigma}_{m}=0, \bar{\Sigma}_{m}= \pm 0.5 \bar{\Sigma}_{m}^{H}$ and $\bar{\Sigma}_{m}= \pm 0.9 \bar{\Sigma}_{m}^{H}$, respectively, where $\bar{\Sigma}_{m}^{H}$ denotes the hydrostatic point delivered by the model MVAR for hydrostatic loading. We precise that we have set $\bar{\Sigma}_{12}=0$. One of the main observation in this figure is the strong dependence of the average deviatoric response on the crystal anisotropy, in the context of cubic systems FCC and BCC. However, even more interestingly, in the porous HCP incompressible (with $K=3$ slip systems) single crystals, the behavior does not evolve from Fig. 10a to Fig. 10c. Hence, while at lower hydrostatic stresses $\left(\left|\bar{\Sigma}_{m}\right| \leq 0.5 \bar{\Sigma}_{m}^{H}\right)$ the HCP porous crystal is more compliant than the FCC and BCC ones, as the pressure increases the FCC and BCC become gradually softer than the HCP. 
a)

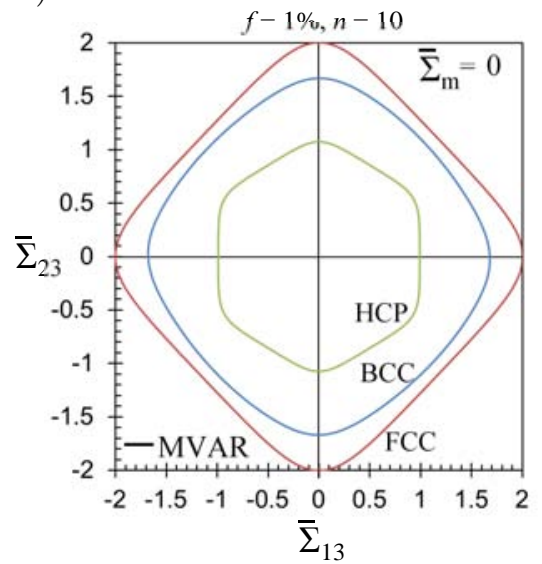

b)

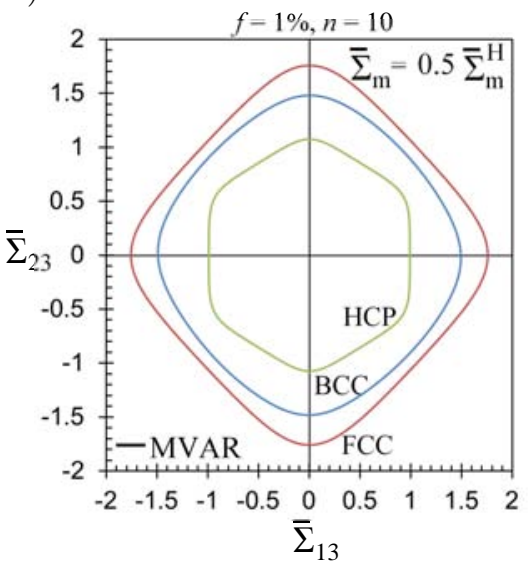

c)

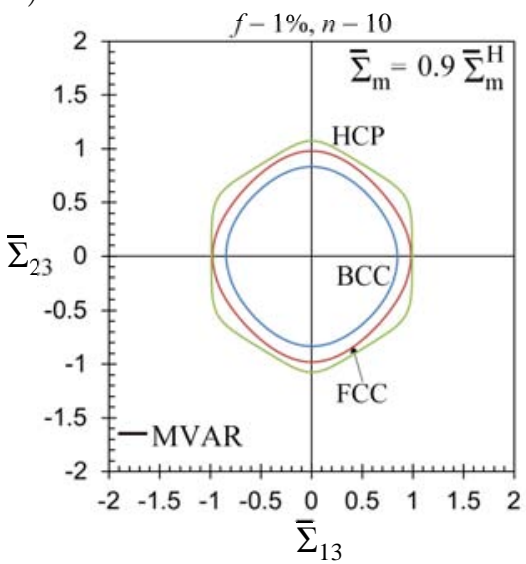

Figure 10: Gauge surfaces in the $\bar{\Sigma}_{13}-\bar{\Sigma}_{23}$ plane HCP (with $K=3$ slip systems), FCC and BCC porous single crystals with spherical voids $\left(w_{1}=w_{2}=1\right)$, a Lode angle $\bar{\theta}=0$, a porosity $f=1 \%$ and a creep exponent $n=10$. Cases of (a) $\bar{\Sigma}_{m}=0$, (b) $\bar{\Sigma}_{m}= \pm 0.5 \bar{\Sigma}_{m}^{H}$, (c) $\bar{\Sigma}_{m}= \pm 0.9 \bar{\Sigma}_{m}^{H}$, where $\bar{\Sigma}_{m}^{H}$ denotes the hydrostatic point.

At this point, it is perhaps helpful to summarize that in the context of highly anisotropic porous single crystals, the purely hydrostatic response is extremely sensitive to the number of the slip systems $K$ as well as on the slip orientations, as clearly shown in Fig. 6, Fig. 9 and Fig. 10 but also confirmed with the FE results in the previous section. These observations have strong implications on void growth and final failure of such single porous crystals. Similar conclusions have also been made recently by Yerra et al. (2010) and Srivastava and Needleman (2015), where the combined effect of crystal orientation and loading directions can lead to the activation of a small or large number of slip systems and subsequently to moderate or significant void growth. Such effects are physically included in the MVAR model as shown in this study. But more detailed analysis of the void growth and shape effects should be included via evolution equations similar to those presented in Danas and Ponte Castañeda (2009a) and Danas and Aravas (2012). Such a work is underway and will be presented elsewhere.

\subsection{Effect of the void shape and orientation}

In this section, we discuss in more detail the effect of microstructure anisotropy upon the effective response of the porous composite. More precisely, Fig. 11 shows MVAR gauge surfaces in the $\bar{\Sigma}_{m}-\bar{\Sigma}_{e q}$ plane for a porous FCC single crystal and a creep exponent $n=10$. The effect of porosity is investigated by choosing $f=(1 \%, 5 \%, 10 \%)$ for different microstructures (a) $w_{1}=w_{2}=1$ and (b) $w_{1}=5, w_{2}=0.2$, $\mathbf{n}^{(1)}=\mathbf{e}^{(1)}, \mathbf{n}^{(2)}=\mathbf{e}^{(2)}$. In Fig. 11, the gauge surfaces exhibit a gradual decrease with increasing porosity for both ellipsoidal $\left(w_{1}=w_{2}=1\right)$ and ellipsoidal $\left(w_{1}=w_{2}^{-1}=5\right)$ voids, as already expected. Nevertheless, while for the case of spherical voids $\left(w_{1}=w_{2}=1\right)$, in Fig. 11a, the curves are symmetric with respect to the $\bar{\Sigma}_{e q}$ axis, the curves for the ellipsoidal voids $\left(w_{1}=w_{2}^{-1}=5\right)$, in Fig. 11b, become asymmetric as already discussed in the context of Fig. 7. As a consequence of this asymmetry, the MVAR estimates are found to be stiffer in the negative pressure regime $\left(\bar{\Sigma}_{m}<0\right)$. The observed asymmetry becomes more pronounced with increasing porosity.

Figure 12 shows gauge surfaces in the $\bar{\Sigma}_{m}-\bar{\Sigma}_{e q}$ plane for a FCC porous single crystal and a creep exponent $n=10$. The porosity of set to $f=5 \%$ and the void orientation is $O_{11}$ (see Table 4 ). The effect of the void aspect ratios is investigated by choosing spherical voids $\left(w_{1}=w_{2}=1\right)$, prolate voids $\left(w_{1}=w_{2}=5\right)$, oblate voids $\left(w_{1}=w_{2}=0.2\right)$ and arbitrary ellipsoidal voids $\left(w_{1}=w_{2}^{-1}=5\right)$. The main observation here is that non-spherical void shapes have an important influence on the effective response of the porous single crystal. Indeed, the slopes of the gauge surfaces depend strongly on the void shape. Specifically, a porous single crystal with ellipsoidal voids $\left(w_{1}=w_{2}^{-1}=5\right)$ is softer than that with oblate voids $\left(w_{1}=w_{2}=0.2\right)$ in the full range of stress triaxialities. Moreover, for the same value of porosity, non-spherical void shapes lead to a significantly more compliant response at high values of the mean stress, especially in the case of oblate and arbitrary ellipsoidal voids. The purely hydrostatic point $\bar{\Sigma}_{m}$ (for $\bar{\Sigma}_{e q}=0$ ) becomes almost double as one goes from the general ellipsoidal voids $\left(w_{1}=w_{2}^{-1}=5\right.$ ) to the spherical ones $\left(w_{1}=w_{2}=1\right)$. It is also evident from this figure that arbitrary ellipsoidal shapes 
a)

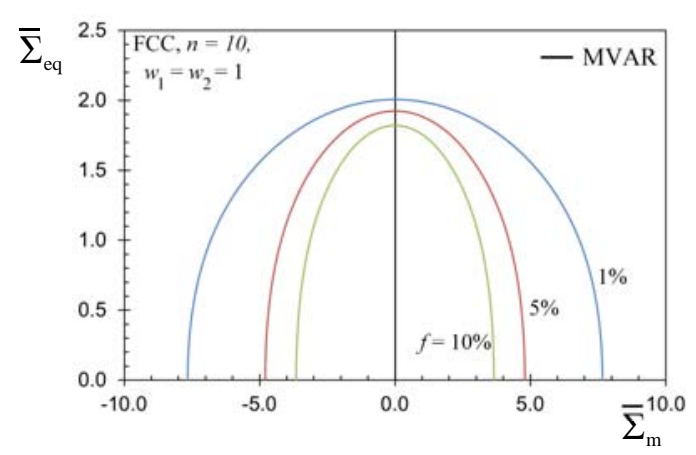

b)

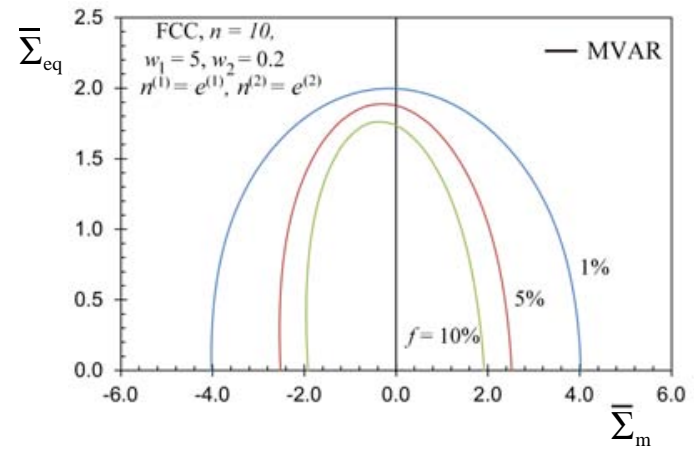

Figure 11: Gauge surfaces in the $\bar{\Sigma}_{m}-\bar{\Sigma}_{e q}$ plane for a FCC porous single crystal with ellipsoidal voids and a creep exponent $n=10$ and a Lode angle $\bar{\theta}=0$. The effect of porosity is investigated by choosing $f=(1 \%, 5 \%, 10 \%)$ for different void shapes (a) $w_{1}=w_{2}=1$ and (b) $w_{1}=5, w_{2}=0.2, \mathbf{n}^{(1)}=\mathbf{e}^{(1)}, \mathbf{n}^{(2)}=\mathbf{e}^{(2)}$.

$\left(w_{1}=w_{2}^{-1}=5\right)$ lead to very different responses when compared with spheroidal shapes $\left(w_{1}=w_{2}=5\right.$ or $w_{1}=w_{2}=0.2$ ).

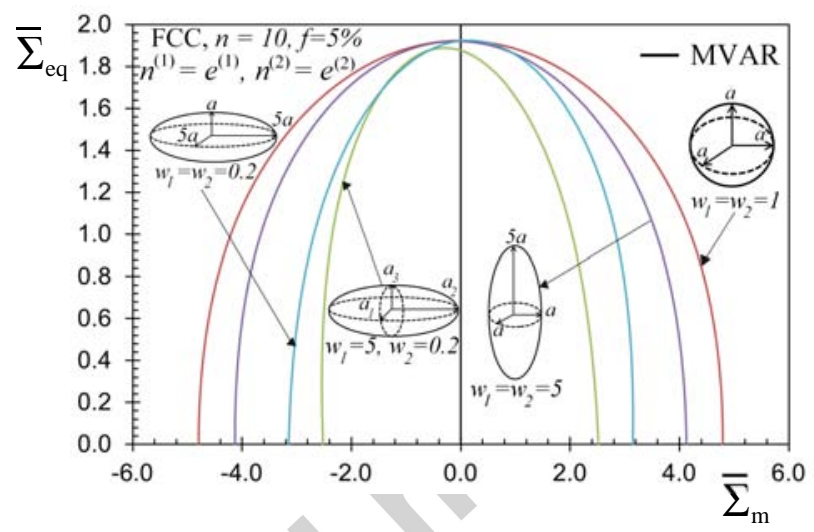

Figure 12: Gauge surfaces in the $\bar{\Sigma}_{m}-\bar{\Sigma}_{e q}$ plane for a FCC porous single crystal with ellipsoidal voids, a Lode angle $\bar{\theta}=0$ and a creep exponent $n=10$. The porosity of set to $f=5 \%$ and the void orientation is $O_{11}$ of Table 4. The effect of the void aspect ratios is investigated by choosing spherical voids $w_{1}=w_{2}=1$, prolate voids $w_{1}=w_{2}=5$, oblate voids $w_{1}=w_{2}=0.2$ and ellipsoidal voids $w_{1}=w_{2}^{-1}=5$.

\begin{tabular}{|c|c|c|c|}
\hline Void Orientation & $\mathbf{n}^{(1)}$ & $\mathbf{n}^{(2)}$ & $\mathbf{n}^{(3)}=\mathbf{n}^{(1)} \times \mathbf{n}^{(2)}$ \\
\hline$O_{11}$ & {$[1,0,0]$} & {$[0,1,0]$} & {$[0,0,1]$} \\
\hline$O_{12}$ & {$[1,0, \overline{1}]$} & {$[0,1,0]$} & {$[1,0,1]$} \\
\hline$O_{13}$ & {$[0,1,0]$} & {$[1,0,0]$} & {$[0,0,1]$} \\
\hline
\end{tabular}

Table 4: Coordinates of the orientations $O_{11}, O_{12}, O_{13}$ (section 6.2) in the reference laboratory frame axes $\mathbf{e}^{(i)}, i=1,2,3$.

Figure 13 shows gauge surfaces in the $\bar{\Sigma}_{m}-\bar{\Sigma}_{e q}$ plane for a FCC porous single crystal and a creep exponent $n=10$. The porosity of set to $f=5 \%$. The effect of the void orientation is adressed by choosing $O_{11}, O_{12}, O_{13}$ (see Table 4) for given aspect ratios $w_{1}=w_{2}^{-1}=w=5$. Note that, as already expected from earlier studies (Danas and Ponte Castañeda, 2009b), the hydrostatic point for all these cases is independent of the void orientation. This is intuitively expected since the hydrostatic loading has no preferential direction (i.e., is isotropic). However, the entire behavior, and in particular the asymmetry with respect to the $\bar{\Sigma}_{e q}$ axis is strongly influenced by the void orientation.

\subsection{Effect of crystal anisotropy and void shape and orientation}

In this section, we investigate the effective response of the porous single crystal in the deviatoric plane $\bar{\Sigma}_{13}-\bar{\Sigma}_{23}$ for given hydrostatic stress $\bar{\Sigma}_{m}$. This allows to probe a more detailed response of the 


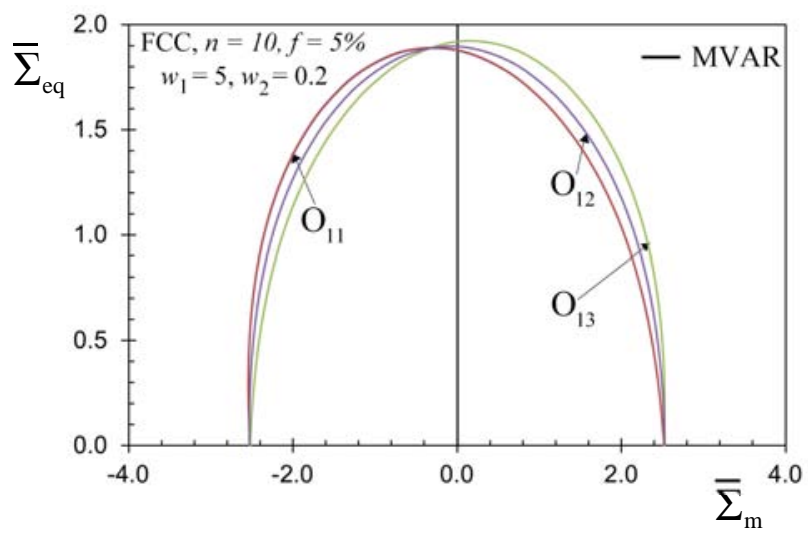

Figure 13: Gauge surfaces in the $\bar{\Sigma}_{m}-\bar{\Sigma}_{e q}$ plane for a FCC porous single crystal with ellipsoidal voids, a Lode angle $\bar{\theta}=0$ and a creep exponent $n=10$. The porosity of set to $f=5 \%$. The effect of the void orientation by choosing $O_{11}, O_{12}, O_{13}$ of Table 4 for given aspect ratios $w_{1}=w_{2}^{-1}=5$.

porous crystal and reveal with clarity the combined coupling between the anisotropy of the crystal and that induced by the void shape and orientation. For the sake of conciseness, in the following, we consider only porous FCC single crystal with porosity $f=5 \%$, void shape $w_{1}=w_{2}^{-1}=5$ and creep exponent $n=10$. The void orientations considered are summarized in Table 5 . In addition, for simplicity, we have set $\bar{\Sigma}_{12}=0$.

\begin{tabular}{|c|c|c|c|}
\hline Orientation & $\mathbf{n}^{(1)}$ & $\mathbf{n}^{(2)}$ & $\mathbf{n}^{(3)}=\mathbf{n}^{(1)} \times \mathbf{n}^{(2)}$ \\
\hline$O_{21}$ & {$[1,0,0]$} & {$[0,1,0]$} & {$[0,0,1]$} \\
\hline$O_{22}$ & {$[1,0,1]$} & {$[0,1,0]$} & {$[1,0,1]$} \\
\hline
\end{tabular}

Table 5: Coordinates of the orientations $O_{21}, O_{22}$ (subsection 6.3) in the reference laboratory frame axes $\mathbf{e}^{(i)}, i=$ $1,2,3$.

In this connection, Fig. 14 shows MVAR cross-sections corresponding to different fixed overall hydrostatic stresses $\bar{\Sigma}_{m}=0, \bar{\Sigma}_{m}= \pm 0.5 \bar{\Sigma}_{m}^{H}, \bar{\Sigma}_{m}= \pm 0.9 \bar{\Sigma}_{m}^{H}$, where $\bar{\Sigma}_{m}^{H}$ denotes the hydrostatic point of the MVAR model for each of the given cases in Fig. 14a,b, respectively.

More specifically, in Fig. 14a, which corresponds to void orientation $O_{21}$ (voids aligned with the principal loading directions), we observe a gradual shrinking of the curves with increasing $\bar{\Sigma}_{m}$ as expected. At small values of $\bar{\Sigma}_{m}=0$, the curve exhibits an almost discrete character which tends to become more rounded (smoothen) with increasing $\bar{\Sigma}_{m}$. Note at this point that since the FCC porous crystal is considered to be rate-dependent (i.e., finite value of the creep exponent $n$ ), the corresponding effective response is strictly convex and the curves exhibit large but finite curvature areas (i.e., smooth vertices) leading to an almost tetragonal symmetry of the curves. When the voids are aligned with the principal loading directions (orientation $O_{21}$ ), the porous crystal preserves the original deviatoric symmetries of the crystal matrix for all values of $\bar{\Sigma}_{m}$ considered. In particular, in this case the curve is fully symmetric with respect to the two axes $\bar{\Sigma}_{13}$ and $\bar{\Sigma}_{23}$.

By contrast, as shown in Fig. 14b, if one considers a non aligned ellipsoidal void oriented as in the case $O_{22}$ in Table 5, the corresponding MVAR curves exhibit an asymmetry with respect to the $\bar{\Sigma}_{13}$-axis, but still preserve the symmetry with respect to the $\bar{\Sigma}_{23}$ axis for $\bar{\Sigma}_{m}>0$. Rather interestingly, the almost tetragonal symmetry still prevails for $\bar{\Sigma}_{m}=0$. This response is a direct consequence of the geometric coupling of the crystal slip orientations and the void shape at finite hydrostatic stresses. Note further that point symmetry of the curves with respect to the global origin $\left(\bar{\Sigma}_{13}, \pm \bar{\Sigma}_{23}\right)=(0,0)$ and $\bar{\Sigma}_{m}=0$ is still preserved. This is easily observed by noting the point symmetries between the continuous lines corresponding to $\bar{\Sigma}_{m}>0$ and the dashed ones for $\bar{\Sigma}_{m}<0$.

Moreover, Fig. 15 displays gauge surfaces in the П-plane (or octahedral plane) corresponding to different fixed overall hydrostatic stresses $\bar{\Sigma}_{m}=0, \bar{\Sigma}_{m}= \pm 0.5 \bar{\Sigma}_{m}^{H}, \bar{\Sigma}_{m}= \pm 0.9 \bar{\Sigma}_{m}^{H}$, where $\bar{\Sigma}_{m}^{H}$ denotes the hydrostatic point of the MVAR model for each of the given cases in Fig. 14a,b, respectively. In these two cases, a gradual shrinking of the curves appears while increasing $\bar{\Sigma}_{m}$, as expected. At small values of $\bar{\Sigma}_{m}=0$, the curve exhibits a quasi discrete character which tends to become more rounded 
a)

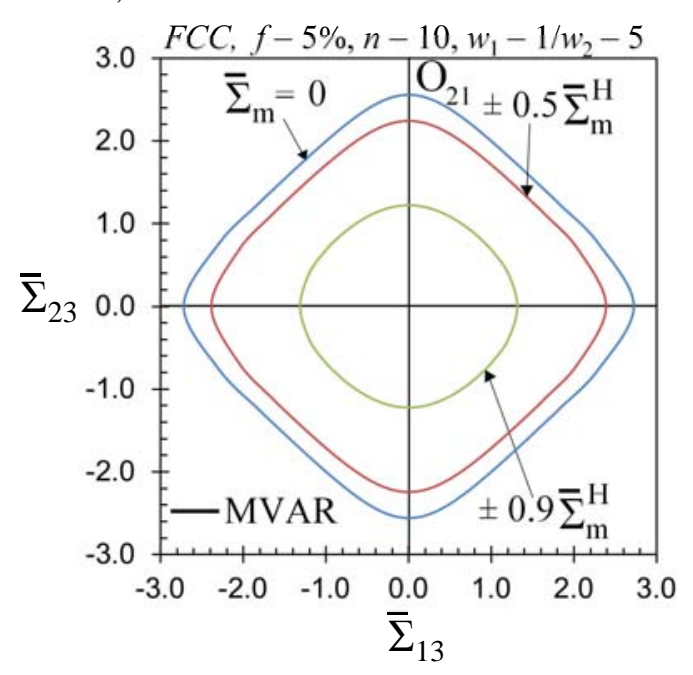

b)

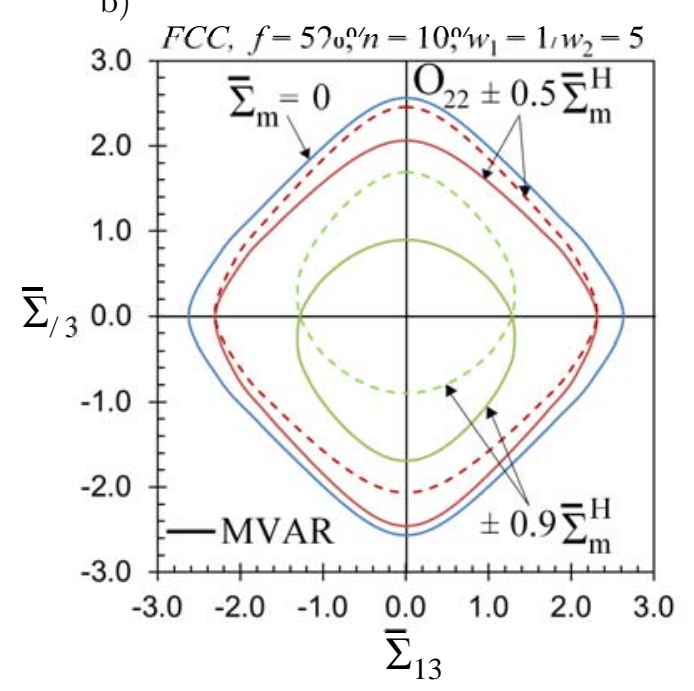

Figure 14: Gauge surfaces in the deviatoric plane $\bar{\Sigma}_{13}-\bar{\Sigma}_{23}$ for a FCC porous single crystal with porosity $f=5 \%$, a Lode angle $\bar{\theta}=0$, void shape $w_{1}=1 / w_{2}=5$ and creep exponent $n=10$, at different level of pressure. The dashed line curves correspond to the negative pressure regime while the continuous one correspond to the positive pressure regime. Case of void orientation (a) $\mathrm{O}_{21}$, (b) $\mathrm{O}_{22}$ of Table 5.

with increasing $\bar{\Sigma}_{m}$. In addition, the gauge surfaces exhibit full asymmetry for finite hydrostatic stresses $\bar{\Sigma}_{m}=0$, but preserve the hexagonal symmetry for $\bar{\Sigma}_{m} \neq 0$. The observed asymmetry is much more pronounced at higher values of $\bar{\Sigma}_{m}$, which is consistent with similar studies in isotropic systems (see for instance Danas et al. (2008b)). Furthermore, it should be stressed that point symmetry of the curves with respect to the global origin and $\bar{\Sigma}_{m}=0$ is still preserved if one compares the continuous $\left(\bar{\Sigma}_{m} \geq 0\right)$ with the dashed lines $\left(\bar{\Sigma}_{m} \leq 0\right)$.

a)

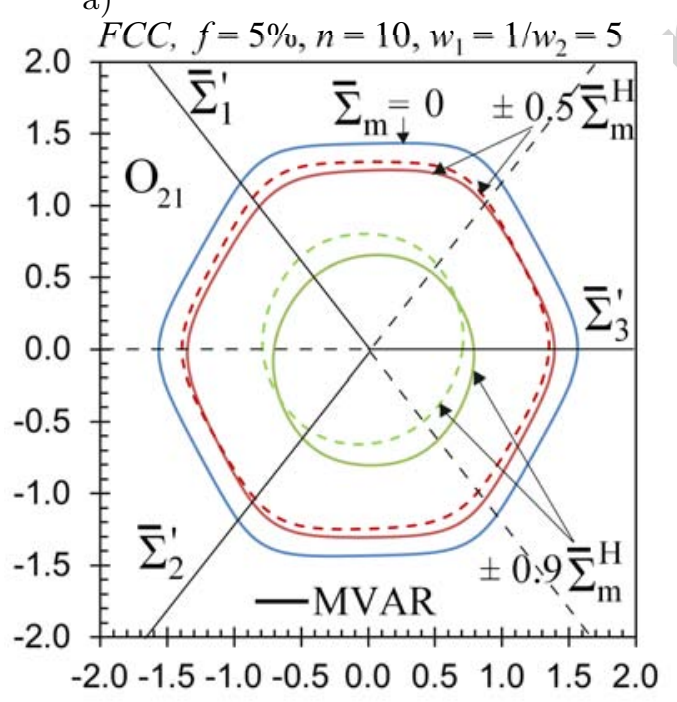

b)

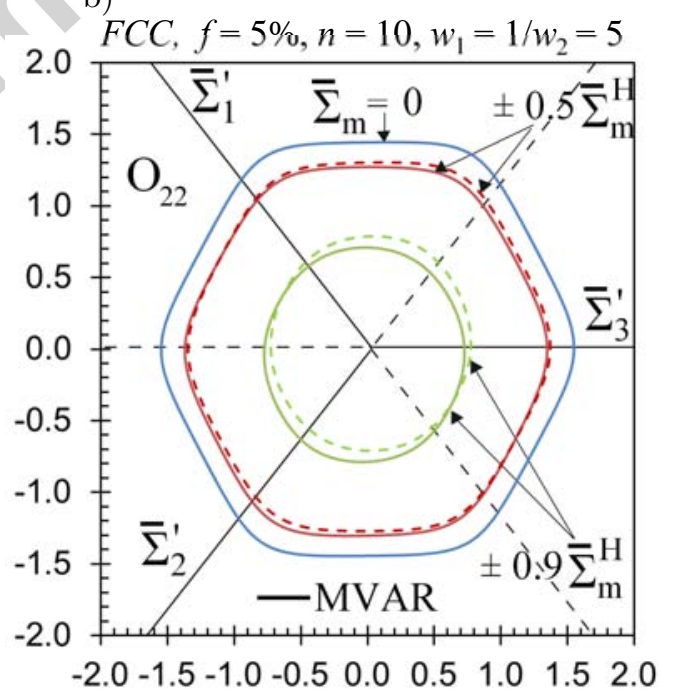

Figure 15: Gauge surfaces in the П-plane (or octahedral plane) for a FCC porous single crystal with porosity $f=5 \%$, a Lode angle $\bar{\theta}=0$, void shape $w_{1}=1 / w_{2}=5$ and creep exponent $n=10$, at different level of pressure. The dashed line curves correspond to the negative pressure regime while the continuous one correspond to the positive pressure regime. Case of void orientation (a) $\mathrm{O}_{21}$, (b) $\mathrm{O}_{22}$ of Table 5 . 


\section{Results - III: Calibration and assessment of the rate-independent MVAR model}

In this section, we investigate the MVAR predictions in the special, albeit very important case, of the rate-independent porous single crystals by considering the limit of the rate sensitivity exponent $n \longrightarrow \infty$. Comparison with existing models in the literature (Han et al. (2013); Paux et al. (2015)) and FE calculations in the context of spherical voids is carried out. Supplementary results for ellipsoidal voids are also shown where the MVAR is compared with FEM. As stated earlier the MVAR gradually underestimates the gauge surface at large nonlinearities. This effect is mainly attributed to the inherent quadratic character of the MVAR model due to the LCC methodology used here, whereas studies in the context of isotropic porous materials have shown that a "cosh" (i.e., exponential) (see for instance the recent study of Cao et al. (2015)) dependence on pressure is more appropriate. However, even if the MVAR model is qualitatively and in most of the cases quantitatively quite accurate (up to $\sim 5 \%$, as seen previously in other cases), the numerical results obtained with FEM in the rate-independent case exhibit a non elliptical shape of the yield surface (see for instance Han et al. (2013)). In order to get better quantitative agreement with the numerical results and still in the context of LCC methods, one needs either to use a more sophisticated approach such as the second-order method (see Ponte Castañeda (2002a,b); Danas et al. (2008a); Danas and Ponte Castañeda (2009a)), the fully numerical variational method (Idiart and Ponte Castañeda, 2007) and iterated variational methods (Agoras and Ponte Castañeda, 2013), or to calibrate the present model which is numerically much easier to be implemented. As will be shown in this section, the best calibration lies somewhere in between the quadratic and "cosh" terms.

\subsection{Calibration of the MVAR}

The MVAR predicts, as expected due to the LCC used in the optimization procedure, a quadratic response for the yield surface. In this regard and motivated by the works of Han et al. (2013), Paux et al. (2015) and Cao et al. (2015), we propose the following simple modification to the original quadratic estimate (43), which becomes

$$
\begin{aligned}
\max _{s=1, K}\left\{\left(\frac{\overline{\boldsymbol{\sigma}} \cdot \boldsymbol{\mu}^{(s)}}{\tau_{0}^{(s)}}\right)^{2}+\frac{f \alpha_{2}}{K\left(\tau_{0}^{(s)}\right)^{2}} \overline{\boldsymbol{\sigma}} \cdot\left(\widehat{\mathbf{S}}^{*}-\mathbf{J} \cdot \widehat{\mathbf{S}}^{*} \mathbf{J}\right) \cdot \overline{\boldsymbol{\sigma}}+\frac{5 \alpha_{1}(1-f)^{2}}{2 K(\ln f)^{2}\left(\tau_{0}^{(s)}\right)^{2}} \overline{\boldsymbol{\sigma}} \cdot \mathbf{J} \cdot \widehat{\mathbf{S}}^{*} \mathbf{J} \cdot \overline{\boldsymbol{\sigma}}+\right. \\
\\
\left.\quad f\left(1-\alpha_{1}\right)\left[\cosh \left(\sqrt{\frac{5}{2 K}} \frac{\cosh ^{-1}\left(\frac{1-f+f^{2}}{f}\right)}{\ln f} \frac{\sqrt{\overline{\boldsymbol{\sigma}} \cdot \mathbf{J} \cdot \widehat{\mathbf{S}}^{*} \mathbf{J} \cdot \overline{\boldsymbol{\sigma}}}}{\tau_{0}^{(s)}}\right)-1\right]-(1-f)^{2}\right\}=0,
\end{aligned}
$$

where $\alpha_{2}$ is a function of the porosity $f$ weighting the relative contribution of the resolved shear stress on each slip system, whereas $\alpha_{1}$ is a parameter introduced to interpolate the shape of the yield surface between an elliptical shape $\left(\alpha_{1}=1\right.$, quadratic) and an exponential "cosh" shape $\left(\alpha_{1}=0\right)$. Moreover, following the result of Ponte Castañeda (2002b) for dilute isotropic porous materials, $\alpha_{2} \sim f^{-1 / 3}$ so that

$$
\frac{\widetilde{\tau}_{0}^{(s)}}{\tau_{0}^{(s)}}-1 \sim 1.5(f / 2)^{2 / 3},
$$

in the dilute limit. The calibration procedure of the above defined coefficients $\alpha_{1}$ and $\alpha_{2}$ leads to the following values

$$
\alpha_{1}=0.65, \quad \alpha_{2} \cong 6 f^{-1 / 3}
$$

It is perhaps interesting to note that the calibration procedure has led to a larger contribution of the original quadratic terms due to the LCC methodology (65\%) than the "cosh" terms (35\%). Moreover, the form of the yield criterion (64) is very similar to the well-known Gurson (1977) model, even though the analysis used here is rather different than the standard limit analysis proposed by the later author. Even so this last model can be thought as a generalization of the Gurson model in the context of porous single crystals and general ellipsoidal void shapes and orientations.

Finally, it should be mentioned that similar modifications as the one in (64) can also be done in the context of the rate-dependent porous single crystals. In those cases, $\alpha_{1}$ becomes readily a function of the rate sensitivity exponent $n$ since at $n=1$ no correction is needed and thus $\alpha=1$ in that case. Nonetheless, due to the fact that the original quadratic MVAR model exhibits good accuracy (max error 
of $\sim 5 \%$ ) for exponents up to $n=10$ such an attempt was not carried out for the rate-independent case in the present work.

\subsection{Comparison with other models}

In this section, the above-described calibrated MVAR model is compared with the models of Han et al. (2013) and Paux et al. (2015), developed for spherical voids in the rate-independent context. In order to assess these models, numerical FE results with complex distribution of voids (see for instance Fig 2a) is used. It is important to precise that for FCC porous single crystals our FE results are quite similar to those obtained by Han et al. (2013) for an FCC crystal with a single-void cubic unit-cell (difference less than $1 \%$ ) for relatively low porosity $f \leq 5 \%$.

Figures 16, 17 and 18 show cross-sections of the effective yield surfaces in the $\bar{\Sigma}_{m}-\bar{\Sigma}_{e q}$ plane for all the different models. Various porosities $f=1 \%, 5 \%$ are considered for both FCC (Fig. 16 and Fig. 17) and BCC crystal structure (Fig. 18). In the context of these figures, we observe a very good agreement between the MVAR predictions and the FE results for the entire range of stress triaxialities. Similarly, Han et al. (2013) and Paux et al. (2015) models are also in very good agreement. In Fig. 17, the principal directions of the stress tensor are oriented along (a) [111] and (b) [210] contrary to most of the figures in the present study that are oriented along the [100] direction. For a more detailed description of this notation the reader is referred to the work of Han et al. (2013). More specifically, for the loading [100] and [111], the MVAR model is in slightly better agreement than the models of Han et al. (2013) and Paux et al. (2015), whereas MVAR tends to slightly underestimate the effective response for the loading $[210]$.

a)

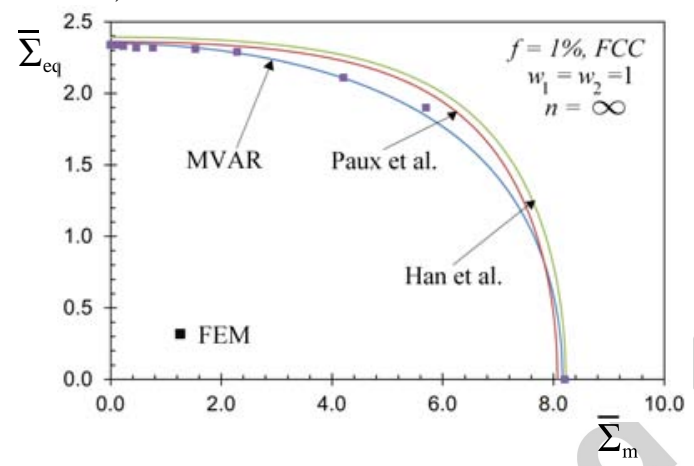

b)

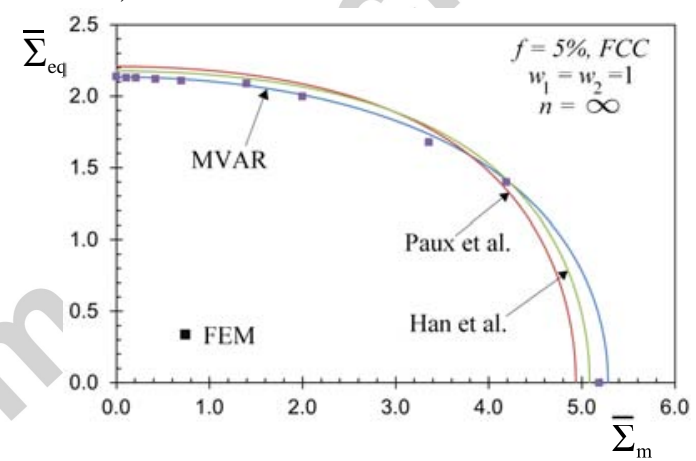

Figure 16: Yield surfaces in the $\bar{\Sigma}_{m}-\bar{\Sigma}_{e q}$ plane for a porous rate independent FCC single crystal comprising spherical voids. Comparison between MVAR model, Han et al. (2013) model, Paux et al. (2015) model and the FE results for a Lode angle $\bar{\theta}=0$ and a porosity (a) $f=1 \%$, (b) $f=5 \%$.

a)

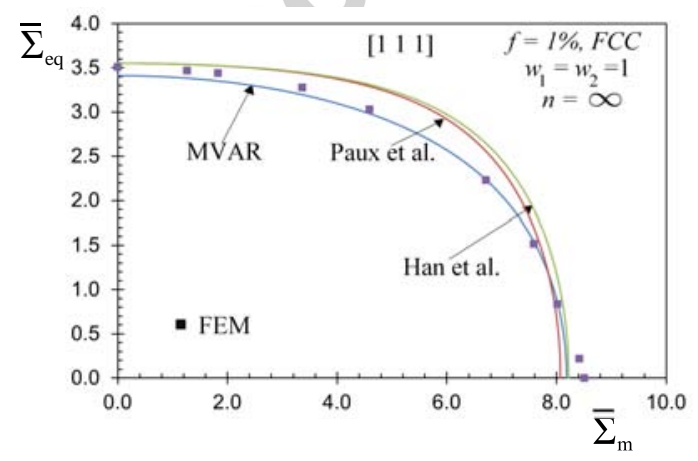

b)

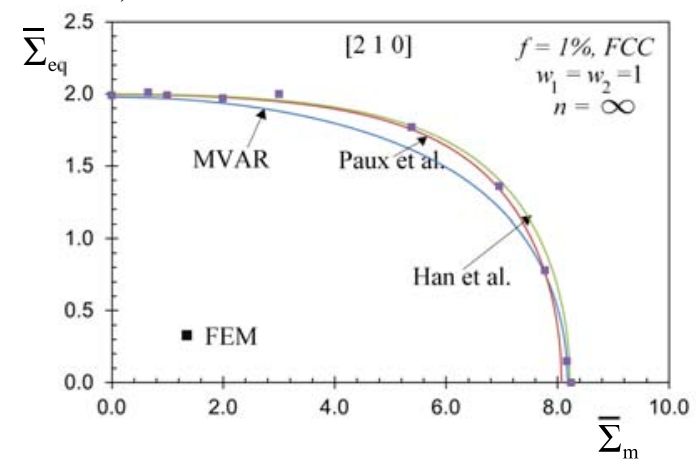

Figure 17: Yield surfaces in the $\bar{\Sigma}_{m}-\bar{\Sigma}_{e q}$ plane for a porous rate independent FCC single crystal comprising spherical voids and porosity $f=1 \%$. Comparison between MVAR model, Han et al. (2013) model, Paux et al. (2015) model and the FE results for Lode angle $\bar{\theta}=0$ where the stress principal directions are oriented along (a) [111] and (b) [210]. The FE results are those in Han et al. (2013). 
a)

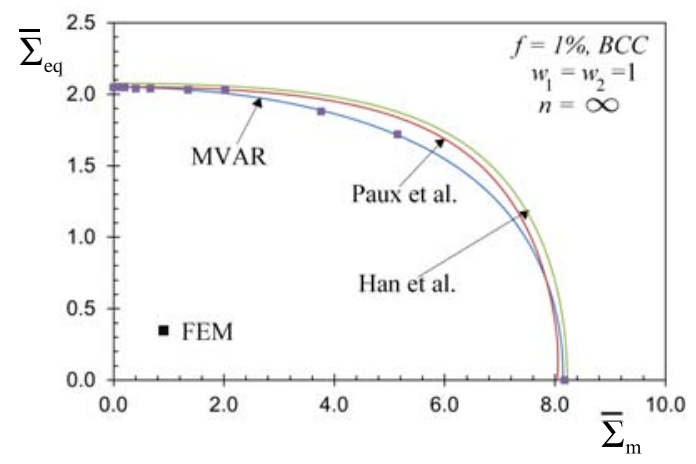

b)

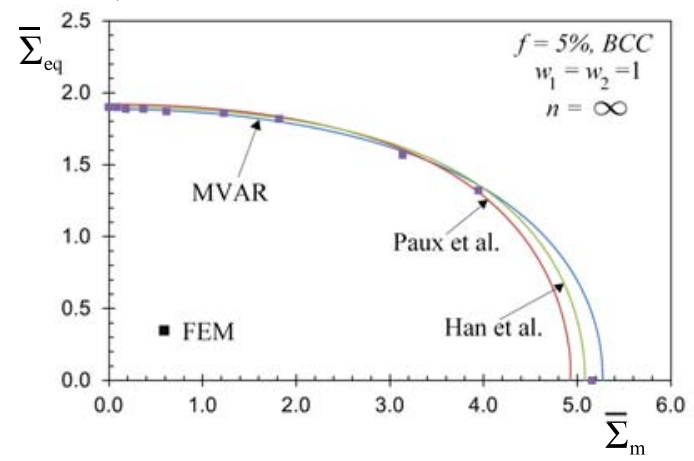

Figure 18: Yield surfaces in the $\bar{\Sigma}_{m}-\bar{\Sigma}_{e q}$ plane for a porous rate independent BCC single crystal comprising spherical voids. Comparison between MVAR model, Han et al. (2013) model, Paux et al. (2015) model and the FE results for a Lode angle $\bar{\theta}=0$ and a porosity (a) $f=1 \%$, (b) $f=5 \%$.

Next, we consider HCP porous single crystals comprising spherical voids with $K=3,6,12$ slip systems. In the case where plastic slip occurs only in the so called basal planes, the slip systems are described by the crystallographic orientations $\{0001\}\langle 1 \overline{2} 10\rangle$ ( $K=3$ slip systems, see Table 3 ). Similar to the rate-dependent results in Fig. 6, when only basal slip systems are considered (i.e., case $C_{1}$ in Table 3), we consider the loading defined in equation (59), whereas for the other two cases $C_{2}$ and $C_{3}$ we apply the loading described in equation (58) (see Table 3).

Then, the computation of the microstructural tensor leads to $\widehat{\mathbf{S}}^{*}=0$, and the MVAR estimate (64) becomes incompressible (see relevant discussion in Section 5), i.e.

$$
\max _{s=1, K}\left\{\left|\overline{\boldsymbol{\sigma}} \cdot \boldsymbol{\mu}^{(s)}\right|-\tau_{0}^{(s)}(1-f)\right\}=0 .
$$

As already seen (see Fig. 6), this prediction is confirmed numerically by FE calculations. In turn, if one considers a crystalline structure with more slip systems such as the case $C_{2}$ with $K=6$ or case $C_{3}$ with $K=12$ slip systems (see Table 3 ), the porous single crystal becomes compressible. In this context, as shown in Fig. 19, an excellent agreement between the MVAR predictions and the FE results for the entire range of stress triaxialities is obtained for both porosities $f=1,5 \%$ used. This result shows clearly that the response at a highly anisotropic porous single crystal is strongly dependent on both the number and the orientation of the slip systems, especially at large hydrostatic stressing. It further suggests that for such low symmetry crystals certain directions appear as "rigid" to plastic deformation thus constrain slip under highly symmetric loads such as purely hydrostatic tension or compression. As a consequence very weak (or even no) void growth is expected in such a case. At this point it should be mentioned that the models of Han et al. (2013) and Paux et al. (2015) have only been calibrated for FCC crystals and therefore at their present form exhibit no such qualitative feature.

Finally, in Fig.20, we show FE results and MVAR predictions for rate-independent porous single crystals comprising ellipsoidal voids, i.e., with aspect ratios $w_{1} \neq 1$ and $w_{2} \neq 1$. The microstructures considered are defined by porosity $f=1 \%$, void orientations $\mathbf{n}^{(1)}=\mathbf{e}^{(1)}, \mathbf{n}^{(2)}=\mathbf{e}^{(2)}, \mathbf{n}^{(3)}=\mathbf{e}^{(3)}$ and two void aspect ratios $w_{1}=w_{2}=(1,4)$ (prolate void). Figure 20a and Fig. 20b present various effective yield surfaces in the $\bar{\Sigma}_{m}-\bar{\Sigma}_{e q}$ plane for FCC and BCC single crystals, respectively. In the context of these figures, the MVAR predictions are in very good agreement with the FE results for several crystal anisotropy types (BCC and FCC) and microstructure anisotropy. For $w_{1}=w_{2}=4$, the yield surfaces exhibit as expected a rather significant "asymmetry" with respect to the $\bar{\Sigma}_{e q}$ axis. This asymmetry, which is strongly sensitive upon the microstructure, is a direct consequence of the coupling between $\bar{\Sigma}_{m}$ and $\bar{\Sigma}_{e q}$ resulting from the complex form of the tensor $\widehat{\mathbf{S}}^{*}$ defined in equation (31). Furthermore, it is interesting to notice that in the positive hydrostatic stress regime $\left(\bar{\Sigma}_{m} \geq 0\right)$ the effective response is softer when the void is more prolate, i.e. when $w_{1}=w_{2}$ increases. In this case, the MVAR model is somewhat less accurate in quantitative terms even if qualitatively it exhibits the correct trends. 
a)

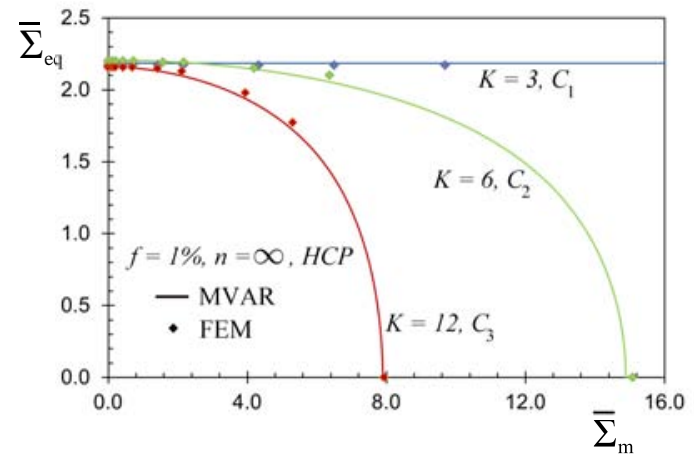

b)

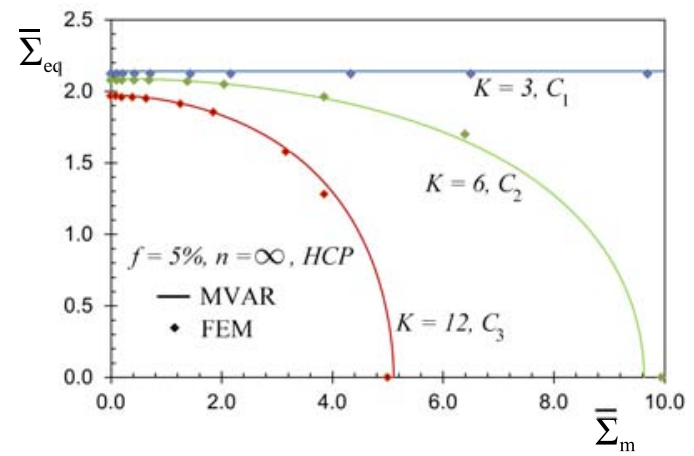

Figure 19: Yield surfaces in the $\bar{\Sigma}_{m}-\bar{\Sigma}_{e q}$ plane for a porous rate independent HCP single crystal comprising spherical voids with $K=3,6,12$ slip systems. Comparison between MVAR model and the FE results for the loading (59) in case $C_{1}$ and the loading (58) and Lode angle $\bar{\theta}=0$ for the cases $C_{2}$ and $C_{3}$. The porosity considered is (a) $f=1 \%$, (b) $f=5 \%$.

a)

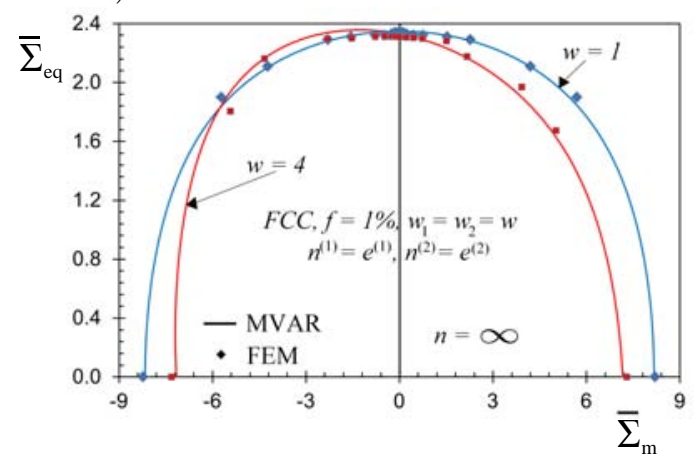

b)

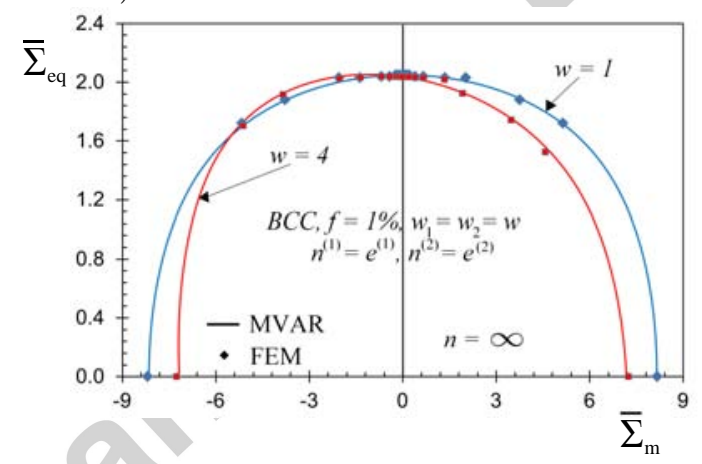

Figure 20: Yield surfaces in the $\bar{\Sigma}_{m}-\bar{\Sigma}_{e q}$ plane for a porous rate independent single crystal comprising ellipsoidal voids of $f=1 \%$, a Lode angle $\bar{\theta}=0$, void orientations $\mathbf{n}_{(1)}=\mathbf{e}_{(1)}, \mathbf{n}_{(2)}=\mathbf{e}_{(2)}, \mathbf{n}_{(3)}=\mathbf{e}_{(3)}$, and two void aspect ratios $w_{1}=w_{2}=(1,4)$. Comparison between the model (MVAR) and the FE results in the cases of (a) FCC, (b) BCC.

\section{Conclusion}

In this study, an analytical constitutive model (MVAR) has been developed for porous rate-dependent and rate-independent single crystals comprising ellipsoidal voids at arbitrary orientation subjected to general loading conditions. In order to achieve this goal, the variational nonlinear homogenization method of Ponte Castañeda (1991a) has been used and modified (Danas and Aravas, 2012) to derive estimates but not bounds. The modified variational (MVAR) model presented in this study, which is an extension of the work of Mbiakop et al. (2015b) in two-dimensions, has been validated by comparison with full field FE calculations of single- and multi-void periodic unit-cells. The MVAR model has been found to be in good agreement with the FE results for a wide range of parameters describing the number and orientation of the slip systems (i.e., crystal anisotropy), the creep exponent (i.e., nonlinearity) of the matrix crystal, the porosity and the void shapes and orientations. The MVAR model has shown strong predictive capabilities while exhibiting critical qualitative features.

More specifically, the MVAR model has been able to predict the strong dependence of the effective response, and especially of the average hydrostatic stress upon the number and orientation of the slip systems as well as the shape and orientation of the voids. One of the major finding of this work, is that for highly anisotropic crystals (e.g., three basal active slip systems in certain HCP crystal structure) the porous crystal can exhibit fully incompressible response, even in the presence of voids. This of course affects the entire effective response of the porous crystal for the entire range of stress states. That is the first time such a result is presented in the literature and reveals the significance of plastic anisotropy of the underlying phases upon the macroscopic response of the material. 
Furthermore, it has been shown that the void shape and orientation affect strongly the response of the porous crystal. In particular, the effective response becomes much softer as one goes from a spherical void to an ellipsoidal one (which is suggestive of a crack-type geometry). In the general case of ellipsoidal voids arbitrarily oriented (with respect to the laboratory axes) and arbitrary crystallographic texture, we have shown that the effective response exhibits no symmetries when plotted in a purely deviatoric plane (and at finite hydrostatic stresses) thus indicating the non-trivial coupling between the anisotropy of the underlying crystal and the (morphological) anisotropy induced by the shape and orientation of the voids.

In this regard, it has been found that the creep exponent has a very strong influence on the effective response of the porous single crystal. Since a large number of technological applications (e.g., single crystals in aerospace industry as well as polycrystalline alloys in nuclear reactors) involve high-temperature and rate-dependent conditions, the MVAR model exhibits promising features that could be important in dealing with such problems. On the other hand, a simple way of calibrating the MVAR model with just two adjustable parameters has been proposed in the rate-independent context so that an excellent agreement with respect to the numerical results is obtained. This calibration procedure can, in the future (if needed), be extended to the rate-dependent case in a straightforward manner. But such an attempt has not been pursued here since the difference between the MVAR estimates and the FE results was found to no more than $\sim 5 \%$ in the worst case studied (i.e., creep exponent $n=10$ ). On the other hand. it should be mentioned that the results shown in the present study, albeit very extensive, do not cover the entire space of loadings and slip orientations as this would make the study extremely long. Hence, the present model should be considered with caution if used in a range of loads or slip system configurations that are very different from those assessed in the present study.

In view of this, we remark that the MVAR model, at this preliminary stage, involves neither dynamic effects, thermal loading nor elasticity effects. Even so, to the best knowledge of the authors, this is the first model in the literature that is able to deal with sufficient accuracy with both rate-dependent and rate-independent porous singe crystals exhibiting general crystal anisotropy, general ellipsoidal void shapes and orientations that are subjected to general three dimensional loading conditions.

Finally, it should be pointed out that several important features present mainly at finite strains, such as lattice rotations, evolution of porosity (e.g., void growth), void shape and orientation are not included in the present analysis (c.f. Danas and Ponte Castañeda (2009b); Danas and Aravas (2012)). These additional features would inevitably lead to well known phenomena in porous materials such as material softening, void shape effects, deformation localization (c.f. Danas and Ponte Castañeda (2012) and Danas and Aravas (2012)) and ultimately void coalescence (see for instance (Benzerga, 2002; Pardoen and Hutchinson, 2000; Yerra et al., 2010; Morin et al., 2015)). Such a work is underway and will be presented elsewhere.

\section{Acknowledgements}

The work of Armel Mbiakop has been supported by a CNRS-INSIS PhD fellowship. Andrei Constantinescu and Kostas Danas greatfully acknowledge partial support by the French National Research Agency (ANR) via the grant INDiANA (ANR-12-RMNP-0011). Kostas Danas also greatfully acknowledges partial support by the French National Research Agency via the grant LOTERIE (ANR-11-BS09-0008).

\section{A. Relation between the single crystal and the isotropic matrix behaviors}

As seen in subsection 2.3, in the special case of slip systems with identical critical resolved shear stress CRSS and reference slip rate $\left(\left(\tau_{0}\right)^{(s)}=\tau_{0},\left(\dot{\gamma}_{0}\right)^{(s)}=\dot{\gamma}_{0}, \forall s=1, K\right)$, the viscoplastic stress potential of the single crystal is written as

$$
U(\boldsymbol{\sigma})=\frac{\dot{\gamma}_{0} \tau_{0}^{-n}}{n+1} \sum_{s=1}^{K}\left|\tau^{(s)}\right|^{n+1} .
$$

If we consider a triaxial loading state, with $\theta$ denoting the Lode angle, the stress tensor can be expressed as

$$
\boldsymbol{\sigma}=\sigma_{m}\{1,1,1\}+\frac{2 \sigma_{e q}}{3}\left\{\cos \theta,-\cos \left(\theta+\frac{\pi}{3}\right),-\cos \left(\theta-\frac{\pi}{3}\right)\right\} .
$$


Moreover, one can parameterized the slip normals and directions of all the slip systems using three Euler angles $\alpha_{1}^{(s)}, \alpha_{2}^{(s)}$ and $\alpha_{3}^{(s)}$, since $\mathbf{m}^{(s)} \perp \mathbf{s}^{(s)}$, such as

$$
\begin{aligned}
\mathbf{m}^{(s)} & =\left(\sin \alpha_{3}^{(s)} \cos \alpha_{1}^{(s)}, \sin \alpha_{3}^{(s)} \sin \alpha_{1}^{(s)}, \cos \alpha_{3}^{(s)}\right)^{T} \\
\mathbf{s}^{(s)} & =\cos \alpha_{2}^{(s)}\left(-\sin \alpha_{1}^{(s)}, \cos \alpha_{1}^{(s)}, 0\right)^{T}+\sin \alpha_{2}^{(s)}\left(\cos \alpha_{3}^{(s)} \cos \alpha_{1}^{(s)}, \cos \alpha_{3}^{(s)} \sin \alpha_{1}^{(s)},-\sin \alpha_{3}^{(s)}\right)^{T}
\end{aligned}
$$

where $0 \leq \alpha_{1}^{(s)}, \alpha_{3}^{(s)} \leq 2 \pi, \quad 0 \leq \alpha_{2}^{(s)} \leq \pi$

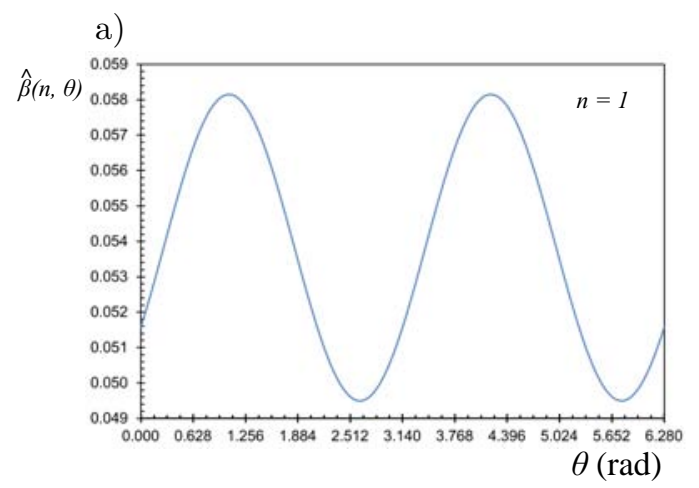

b)

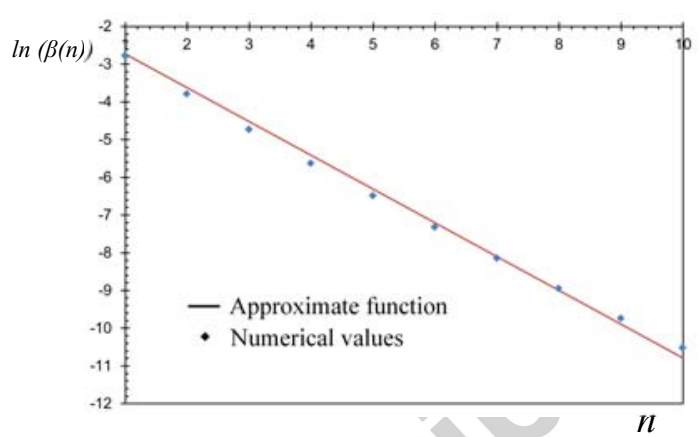

Figure 21: (a) $\widehat{\beta}(n, \theta)$ represented as a function of the Lode angle $\theta$ for a creep exponent $n=1$, (b) Representation of the numerical values and the approximate function of $\beta(n)$ for a range of creep exponents.

Thus, in the limiting case of isotropic matrix, i.e. $K \longrightarrow \infty$, the relations (5), (68), (69) and (70) lead to the following form of the potential

$$
U(\boldsymbol{\sigma}) \cong \frac{\dot{\gamma}_{0} \tau_{0}^{-n}}{n+1} \sigma_{e q}^{n+1} K \lim _{K \rightarrow \infty} \frac{1}{K} h\left(\alpha_{1}^{(s)}, \alpha_{2}^{(s)}, \alpha_{3}^{(s)}, \theta, n\right) \cong \frac{\dot{\gamma}_{0} \tau_{0}^{-n}}{n+1} \sigma_{e q}^{n+1} K \widehat{\beta}(n, \theta)
$$

The numerical computation of $\widehat{\beta}(n, \theta)$ for various values of the Lode angle and a large range of creep exponents exhibits a behavior that can be approximated as periodic function of $\theta$ (see for instance fig $21 \mathrm{a}$, in the case of $n=1$ ). More precisely, one can write

$$
U(\boldsymbol{\sigma}) \cong \frac{\dot{\gamma}_{0} \tau_{0}^{-n}}{n+1} \sigma_{e q}^{n+1} K \widehat{\beta}(n, \theta), \quad \widehat{\beta}(n, \theta) \cong \beta_{n} g_{\theta} \cong \frac{4}{25} 6^{-\frac{n}{2}}\left(\cos \left(\theta-\frac{\pi}{3}\right)\right)^{n+1}
$$

A representation of $\beta_{n}$ is shown in fig $21 \mathrm{~b}$.

\section{B. Homogenized model for the porous single crystal}

In this section, we discuss in more detail the optimization operation in equation (26) as well as the evaluation of the effective energy of the linear comparison composite (LCC) and optimization operation in equation (27). We remind the reader that the goal of the present study is to propose simple analytical models but not bounds. This allows for the relaxation of certain optimization (sup or inf) procedures, as is discussed in the following.

The optimization procedure of the corrector functions $v\left(\lambda^{(s)}\right)$ in $(22)$ is carried out following the work of DeBotton and Ponte Castañeda (1995). First, we consider the limits $\rho \rightarrow \infty$ and $\kappa \rightarrow \infty$ in the linear stress potential of the matrix phase $U_{L}$ (c.f. equation (25)). Then, using relation (9), one finds

$$
U_{L}\left(\boldsymbol{\sigma}, \lambda^{(s)}\right)=\sum_{s=1}^{K} \Psi_{L}^{(s)}\left(\tau^{(s)}, \lambda^{(s)}\right)=\sum_{s=1}^{K} \frac{1}{2 \lambda^{(s)}}\left(\tau^{(s)}\right)^{2}
$$

Next, substituting equations (10) and (73) in (22), the corrector function becomes (Idiart and Ponte Castañeda, 
2007)

$$
\begin{aligned}
v\left(\lambda^{(s)}\right) & =\sup _{\widehat{\sigma}} \sum_{s=1}^{K}\left[\Psi_{L}^{(s)}\left(\widehat{\tau}^{(s)}, \lambda^{(s)}\right)-\Psi^{(s)}\left(\widehat{\tau}^{(s)}\right)\right] \\
& \leq \sum_{s=1}^{K} \sup _{\widehat{\tau}^{(s)}}\left[\frac{1}{2 \lambda^{(s)}}\left(\widehat{\tau}^{(s)}\right)^{2}-\frac{\dot{\gamma}_{0}^{(s)} \tau_{0}^{(s)}}{n+1}\left(\frac{\left|\widehat{\tau}^{(s)}\right|}{\tau_{0}^{(s)}}\right)^{n+1}\right] \\
& \simeq \sum_{s=1}^{K} \operatorname{stat}_{\widehat{\tau}^{(s)}}\left[\frac{1}{2 \lambda^{(s)}}\left(\widehat{\tau}^{(s)}\right)^{2}-\frac{\dot{\gamma}_{0}^{(s)} \tau_{0}^{(s)}}{n+1}\left(\frac{\left|\widehat{\tau}^{(s)}\right|}{\tau_{0}^{(s)}}\right)^{n+1}\right] \\
& =\frac{n-1}{n+1} \frac{\dot{\gamma}_{0}^{(s)} \tau_{0}^{(s)}}{2}\left(\frac{\tau_{0}^{(s)}}{\lambda^{(s)} \dot{\gamma}_{0}^{(s)}}\right)^{\frac{n+1}{n-1}} .
\end{aligned}
$$

In the above equation, following (Idiart and Ponte Castañeda, 2007), we have interchanged the "sup" operation with the summation in order to be able to have an analytical evaluation of the corrector function $v$. Subsequently, we have also relaxed the "sup" to "stat" since the interest in this work is on estimates and no attempt to obtain bounds is made, as already stated in the context of equation (26).

Next, starting from the definition of the stress potential of the matrix phase in the LCC in equation (25), and using the generalized Hashin-Shtrikman estimates (Hashin and Shtrikman (1963)) of Willis (Willis (1977)) which are known to be quite accurate for porous random systems, up to moderate concentrations of pores, one gets

$$
\widetilde{U}_{L}\left(\overline{\boldsymbol{\sigma}}, \mathbf{S} ; s_{\alpha}\right)=\frac{1}{2(1-f)} \overline{\boldsymbol{\sigma}} \cdot\left(\sum_{s=1}^{K} \frac{1}{2 \lambda^{(s)}} \mathbf{E}^{(s)}+f \mathbf{S}^{*}\right) \cdot \overline{\boldsymbol{\sigma}},
$$

where

$$
\mathbf{S}^{*}=\lim _{\rho \rightarrow \infty} \lim _{\kappa \rightarrow \infty} \mathbf{Q}^{-1}-\sum_{s=1}^{K} \frac{1}{2 \lambda^{(s)}} \mathbf{E}^{(s)}, \quad \mathbf{Q}=\mathbf{S}^{-1}-\mathbf{S}^{-1} \cdot \mathbf{P} \cdot \mathbf{S}^{-1} .
$$

The $\mathbf{P}$ is a fourth-order microstructural tensor related to the Eshelby tensor (Eshelby, 1957) and is given by (Willis, 1977)

$$
\mathbf{P}_{i j k l}=\frac{1}{4 \pi \operatorname{det} \boldsymbol{Z}} \int_{|\xi|=1} \frac{\left.\left(\mathbf{S}_{i a k b}^{-1} \xi_{a} \xi_{b}\right)^{-1} \xi_{j} \xi_{l}\right|_{(i j)(k l)}}{\left|\boldsymbol{Z}^{-1} \cdot \xi\right|^{3}} d S .
$$

where $\boldsymbol{Z}$ is given in relation (34) and $\xi$ is a three-dimensional unit-vector. The computation of $\mathbf{Q}$ in the above expressions is critical for the optimization problem (27). Due to the extremely complicated dependence of $\mathbf{Q}$ on $\lambda^{(s)}$, the optimization problem for the estimation of the effective response in equation (27) has to be carried out numerically, except in the special case where we let $\lambda^{(s)} \rightarrow \lambda$ for all $s=1, K$. This simplifies tremendously the evaluation of the $\mathbf{Q}$ tensor and allows for the following approximations. More specifically, in the limit of $\lambda^{(s)} \rightarrow \lambda$, one can easily show that

$$
\lim _{\lambda^{(s)} \rightarrow \lambda} \mathbf{S}=\frac{1}{\lambda} \mathcal{S}(\widehat{\rho}, \widehat{\kappa}), \quad \lim _{\lambda^{(s)} \rightarrow \lambda} \mathbf{P}=\frac{1}{\lambda} \widehat{\mathbf{P}}(\widehat{\rho}, \widehat{\kappa}), \quad \lim _{\lambda^{(s)} \rightarrow \lambda} \mathbf{Q}=\lambda \widehat{\mathbf{Q}}(\widehat{\rho}, \widehat{\kappa})
$$

which implies

$$
\lim _{\lambda^{(s)} \rightarrow \lambda} \mathbf{S}^{*}\left(\lambda^{(s)}\right)=\frac{1}{\lambda} \widehat{\mathbf{S}}^{*}(\widehat{\rho}, \widehat{\kappa}) .
$$

In the above expressions, $\widehat{\rho}=\rho / \lambda$ and $\widehat{\kappa}=\kappa / \lambda$, whereas $\widehat{\mathbf{S}}, \widehat{\mathbf{Q}}, \mathcal{S}$ and $\widehat{\mathbf{P}}$ are independent of $\lambda$ and have been defined in equations (31), (32) and (33), respectively.

Next, following the work of Mbiakop et al. (2015b), we introduce the approximation

$$
\mathbf{S}^{*}\left(\lambda^{(s)}\right) \cong \frac{1}{K} \sum_{s=1}^{K} \frac{1}{\lambda^{(s)}} \widehat{\mathbf{S}}^{*},
$$

where $\widehat{\mathbf{S}}^{*}$ is given by (31). This last expression (80) is identically true for $\lambda^{(s)}=\lambda, \forall s=1, K$ while being 
a relatively good approximation in the neighborhood of $\lambda^{(i)} \simeq \lambda^{(j)}, \forall i, j=1, K$. The choice (80) allows for a fully analytical resolution of the optimization procedure (27) and thus to a fully analytical model. Nonetheless, this approximation is expected to be less accurate in cases where the critical resolved shear stresses of the slip systems are very different.

Consequently, substitution of (74), (75) and (80) in (27) leads to

$\widetilde{U}_{v a r}\left(\overline{\boldsymbol{\sigma}} ; s_{\alpha}\right)=\operatorname{stat}_{\lambda^{(s)} \geq 0}\left[\sum_{s=1}^{K}\left\{\frac{1}{1-f} \frac{1}{2 \lambda^{(s)}}\left(\left(\overline{\boldsymbol{\sigma}} \cdot \boldsymbol{\mu}^{(s)}\right)^{2}+\frac{f}{K} \overline{\boldsymbol{\sigma}} \cdot \widehat{\mathbf{S}}^{*} \cdot \overline{\boldsymbol{\sigma}}\right)\right\}-(1-f) \sum_{s=1}^{K} \frac{n-1}{n+1} \frac{\dot{\gamma}_{0}^{(s)} \tau_{0}^{(s)}}{2}\left(\frac{\tau_{0}^{(s)}}{\lambda^{(s)} \dot{\gamma}_{0}^{(s)}}\right)^{\frac{(n+1)}{(n-1)}}\right]$.

Again, in order to keep the discrete character of the slip system response at purely deviatoric loads and zero porosity as well as analytical tractability of the model, the order of the maximisation can be commuted with the summation (see Han et al. (2013) and Mbiakop et al. (2015b)), such that

$$
\frac{1}{2 \lambda^{(s)}}=\frac{\dot{\gamma}_{0}^{(s)}}{2 \tau_{0}^{(s)}}\left\{\frac{\left(\overline{\boldsymbol{\sigma}} \cdot \boldsymbol{\mu}^{(s)}\right)^{2}+\frac{f}{K} \overline{\boldsymbol{\sigma}} \cdot \widehat{\mathbf{S}}^{*} \cdot \overline{\boldsymbol{\sigma}}}{\left(\tau_{0}^{(s)}\right)^{2}(1-f)^{2}}\right\}^{(n-1) / 2}
$$

Note, however, that the commutative operation in (81) could lead, in general, to an artificial vertex-like response, particularly at very large creep exponents (e.g., $n>50$ ). In any case, we show in the main text that the above procedure leads to rather accurate results in a very large range of creep-exponents $(n=1,2,5,10, \infty)$ for the range of loadings and slip systems considered. Nevertheless, these results do not cover the entire loading or slip orientation space and hence the present model should be considered with caution if used in a range of loads or slip system configurations that are very different from those assessed in the present study.

Finally, substitution of (82) in (81), we get the variational estimate of the effective stress potential of the crystalline porous material as presented in equations (28) or equivalently (29).

\section{References}

Dassault Systems Abaqus. ABAQUS/Standard Version 6.9, user manual. Simulia Corp., 2009.

M. Agoras and P. Ponte Castañeda. Iterated linear comparison bounds for viscoplastic porous materials with ellipsoidal microstructures. J. Mech. Phys. Solids, 61:701-725, 2013.

J.S. Amelang, G.N. Venturini, and D.M. Kochmann. Summation rules for a fully nonlocal energy-based quasicontinuum method. Journal of the Mechanics and Physics of Solids, page doi:10.1016/j.jmps.2015.03.007, 2015.

N. Aravas. Finite elastoplastic transformations of transversely isotropic metals. Int. J. Solids Struct., 29:2137-2157, 1992.

N. Aravas and P. Ponte Castañeda. Numerical methods for porous metals with deformation-induced anisotropy. Comput. Methods Appl. Mech. Engrg., 193:3767-3805, 2004.

I. Barsoum and J. Faleskog. Rupture mechanisms in combined tension and shear- micromechanics. Int. J. Solids Struct., 44:5481-5498, 2007.

A. Benallal. On some features of the effective behavior of porous solids with general incompressible matrix behavior. Comptes Rendus Mécanique, (submitted), 2015.

A. Benallal, R. Desmorat, and M. Fournage. An assessment of the role of the third stress invariant in the gurson approach for ductile fracture. European Journal of Mechanics - A/Solids, 47(0): 400 - 414, 2014. ISSN 0997-7538. doi: http://dx.doi.org/10.1016/j.euromechsol.2014.02.009. URL http://www.sciencedirect.com/science/article/pii/S099775381400028X.

A. Benzerga and J.B. Leblond. Ductile fracture by void growth to coalescence. Adv. Appl. Mech., 44:169-305, 2010.

A. Benzerga, J. Besson, and A. Pineau. Anisotropic ductile fracture. part ii: Theory. Acta Metall., 52:4639-4650, 2004.

A.A. Benzerga. Micromechanics of coalescence in ductile fracture. Journal of the Mechanics and Physics of Solids, 50(6):1331 - 1362, 2002. ISSN 0022-5096. doi: http://dx.doi.org/10.1016/S0022-5096(01)00125-9. URL http://www.sciencedirect.com/science/article/pii/S0022509601001259.

B. Budiansky, J. W. Hutchinson, and S. Slutsky. Void growth and collapse in viscous solids. Mechanics of Solids, The Rodney Hill 60th aniversary Volume, Hopkins, H. G. and Sewell, M. J. eds. Pergamon Press Oxford, pages 13-45, 1982.

T.S. Cao, M. Maziere, K. Danas, and J. Besson. A model for ductile damage prediction at low stress triaxialities incorporating void shape change and void rotation (in revision). Int. J. Solids Struct., 2015.

O. Cazacu, B. Revil-Baudard, N. Chandola, and D. Kondo. New analytical criterion for porous solids with tresca matrix under axisymmetric loadings. Int. J. Solids Struct., 51:861-874, 2014.

E. Combaz, C. Bacciarini, R. Charvet, W. Dufour, and A. Mortensen. Multiaxial yield behaviour of al replicated foam. J. Mech. Phys. Solids, 59:1777-1793, 2011.

K. Danas and N. Aravas. Numerical modeling of elasto-plastic porous materials with void shape effects at finite deformations. Composites: Part B, 43(2544-2559), 2012.

K. Danas and P. Ponte Castañeda. A finite-strain model for anisotropic viscoplastic porous media: I-theory. Eur. J. Mech. A/Solids, 28:387-401, 2009a. 
K. Danas and P. Ponte Castañeda. A finite-strain model for anisotropic viscoplastic porous media: Ii-applications. Eur. J. Mech. A/Solids, 28:402-416, 2009b.

K. Danas and P. Ponte Castañeda. Influence of the lode parameter and the stress triaxiality on the failure of elasto-plastic porous materials. International Journal of Solids and Structures, 49(11âa'c12): 1325 - 1342, 2012. ISSN 0020-7683. doi: http://dx.doi.org/10.1016/j.ijsolstr.2012.02.006. URL http://www.sciencedirect.com/science/article/pii/S0020768312000467.

K. Danas, M. Idiart, and P. Ponte Castañeda. A homogenization-based constitutive model for two-dimensional viscoplastic porous media. C. R. Mecanique, 336:79-90, 2008a.

K. Danas, M.I. Idiart, and P. Ponte Casta neda. A homogenization-based constitutive model for isotropic viscoplastic porous media. International Journal of Solids and Structures, 45(11â í"12): 3392 - 3409, 2008b. ISSN 0020-7683. doi: http://dx.doi.org/10.1016/j.ijsolstr.2008.02.007. URL http://www.sciencedirect.com/science/article/pii/S0020768308000656.

G. DeBotton and P. Ponte Castañeda. Variational estimates for the creep behaviour of polycrystals. Proceedings of the Royal Society of London A: Mathematical, Physical and Engineering Sciences, 448(1932):121-142, 1995. ISSN 0962-8444. doi: 10.1098/rspa.1995.0009.

M. Dunand and D. Mohr. Effect of lode parameter on plastic flow localisation after proportional loading at low stress triaxialities. J. Mech. Phys. Solids, 66:133-153, 2014.

J.D. Eshelby. The determination of the elastic field of an ellipsoidal inclusion and related problems. Proc. R. Soc. Lond. A, 241:376-396, 1957.

L. Flandi and J.-B. Leblond. A new model for porous nonlinear viscous solids incorporating void shape effects-i: Numerical validation. Eur. J. Mech. A/Solids, 24:552-571, 2005.

Felix Fritzen, Samuel Forest, Thomas BÃhlke, Djimedo Kondo, and Toufik Kanit. Computational homogenization of elasto-plastic porous metals. International Journal of Plasticity, 29(0):102 - 119, 2012. ISSN 0749-6419. doi: http://dx. doi.org/10.1016/j.ijplas.2011.08.005. URL http://www.sciencedirect.com/science/article/pii/S0749641911001458.

Y. Gan and J. Kysar. Cylindrical void in a rigid-ideally plastic single crystal iii: hexagonal close-packed crystal. Int. J. Plasticity, 23:592-619, 2007.

Y. Gan, J. Kysar, and T. Morse. Cylindrical void in a rigid-ideally plastic single crystal ii: experiments and simulations. Int. J. Plasticity, 22:39-72, 2006.

P. Gilormini and J.-C Michel. Finite element solution of the problem of a spherical inhomogeneity in an infinite power-law viscous matrix. Eur. J. Mech. A/Solids, 17:725-740, 1998.

A.L. Gurson. Continuum theory of ductile rupture by void nucleation and growth. J. Eng. Mater. Tech., 99:2-15, 1977.

S. Ha and K. Kim. Void growth and coalescence in f.c.c. single crystals. Int. J. Solids Struct., 52:863-873, 2010.

X. Han, J. Besson, S. Forest, B. Tanguy, and S. Bugat. A yield function for single crystals containing voids. International Journal of Solids and Structures, 50:2115-2131, 2013.

Z. Hashin and S. Shtrikman. A variational approach to the theory of the elastic behaviour of multiphase materials. $J$. Mech. Phys. Solids, 11:127-140, 1963.

E. Herve and A. Zaoui. n-layered inclusion-based micromechanical modelling. Int. J. Engng Sci., 31:1-10, 1993.

R. Hill. Elastic properties of reinforced solids: Some theoritical principles. J. Mech. Phys. Solids, 11:357-372, 1963.

M. Huang, Z. Li, and C. Wang. Discrete dislocation dynamics modelling of microvoid growth and its intrinsic mechanism in single crystals. Acta Mater., 55:1387-1396, 2007.

M. Huang, L. Zhao, and J. Tong. Discrete dislocation dynamics modelling of mechanical deformation of nickel-based single crystal superalloys. Int. J. Plasticity, 28:141-158, 2012.

Y. Huang. A user material subroutine incorporating single crystal plasticity in the abaqus finite element program. Mech Report, Division of Engineering and Aplied Sciences, Harvard University, 178, 1991.

M. Hussein, U. Borg, C. Niordson, and V. Deshpande. Plasticity size effects in voided crystals. J. Mech. Phys. Solids, 56: 114-131, 2008.

M. Idiart and P. Ponte Castañeda. Variational linear comparison bounds for nonlinear composites with anisotropic phases. ii. crystalline materials. Proc. R. Soc. A, 463:925-943, 2007.

M.I. Idiart, H. Moulinec, P. Ponte Casta neda, and P. Suquet. Macroscopic behavior and field fluctuations in viscoplastic composites: Second-order estimates versus full-field simulations. Journal of the Mechanics and Physics of Solids, 54(5):1029 - 1063, 2006. ISSN 0022-5096. doi: http://dx.doi.org/10.1016/j.jmps.2005.11.004. URL http://www.sciencedirect.com/science/article/pii/S0022509605002188.

F.P Jimenez and S. Pellegrino. Constitutive modeling of fiber composites with a soft hyperelastic matrix. International Journal of Solids and Structures, 49:635-647, 2012.

M. Kailasam and P. Ponte Castañeda. A general constitutive theory for linear and nonlinear particulate media with microstructure evolution. J. Mech. Phys. Solids, 46:427-465, 1998.

S.M. Keralavarma, S. Hoelscher, and A.A. Benzerga. Void growth and coalescence in anisotropic plastic solids. Int. J. Solids Struct., 48:1696-1710, 2011.

B. Kondori and A.A. Benzerga. Fracture strains, damage mechanisms and anisotropy in a magnesium alloy across a range of stress triaxialities. Experimental Mechanics, 54(3):493-499, 2014a. ISSN 0014-4851. doi: 10.1007/s11340-013-9812-8. URL http://dx.doi.org/10.1007/s11340-013-9812-8.

Babak Kondori and A.Amine Benzerga. Effect of stress triaxiality on the flow and fracture of mg alloy az31. Metallurgical and Materials Transactions A, 45(8):3292-3307, 2014b. ISSN 1073-5623. doi: 10.1007/s11661-014-2211-7. URL http://dx.doi.org/10.1007/s11661-014-2211-7.

J. Kysar, Y. Gan, and G. Mendez-Arzuza. Cylindrical void in a rigid-ideally plastic single crystal. part i: Anisotropic slip line theory solution for face-centered cubic crystals. Int. J. Plasticity, 21:1481-1520, 2005.

J.W. Kysar. Addendum to 'a user material subroutine incorporating single crystal plasticity in the abaqus finite element program'. Mech Report, Division of Engineering and Aplied Sciences, Harvard University, 178, 1997.

J. Leblond, G. Perrin, and P. Suquet. Exact results and approximate models for porous viscoplastic solids. Int. J. Plasticity, 10:213-235, 1994.

F. López Jiménez. Modeling of soft composites under three-dimensional loading. Composites: Part B, 59:173-180, 2014.

Oscar Lopez-Pamies, Taha Goudarzi, and Kostas Danas. The nonlinear elastic response of suspensions of rigid inclusions 
in rubber: II - a simple explicit approximation for finite-concentration suspensions. Journal of the Mechanics and Physics of Solids, 61(1):19 - 37, 2013. ISSN 0022-5096. doi: http://dx.doi.org/10.1016/j.jmps.2012.08.013. URL http://www.sciencedirect.com/science/article/pii/S0022509612001925.

J. Mandel. Contribution théorique à létude de lécrouissage et des lois d'écoulement plastique. In 11th International Congress on Applied mechanics, 1964.

J. Marshall and K. Dayal. Atomistic-to-continuum multiscale modeling with long-range electrostatic interactions in ionic solids. J. Mech. Phys. Solids, 62:137-162, 2014.

A. Mbiakop, A. Constantinescu, and K. Danas. On void shape effects of periodic elasto-plastic materials subjected to cyclic loading. European Journal of Mechanics and Solids, 49:481-499, 2015a.

A. Mbiakop, A. Constantinescu, and K. Danas. A model for porous single crystals with cylindrical voids of elliptical cross-section (submitted). Int. J. Solids Struct., page in press, 2015b.

F. Mc Clintock. A criterion for ductile fracture by the growth of holes. J. App. Mech., 35:363-371, 1968.

J.-C. Michel, H. Moulinec, and P. Suquet. Effective properties of composite material with periodic microstructure: a computational approach. Comput. Methods Appl. Mech. Engrg., 172:109-143, 1999.

J.C. Michel and P. Suquet. The constitutive law of nonlinear viscous and porous materials. J. Mech. Phys. Solids, 40: 783-812, 1992.

C. Miehe, J. Schroder, and J. Schotte. Computational homogenization analysis in finite plasticity simulation of texture development in polycrystalline materials. Computer Methods in Applied Mechanics and Engineering, 171:387-418, 1999.

V. Monchiet, O. Cazacu, E. Charkaluk, and D. Kondo. Macroscopic yield criteria for plastic anisotropic materials containing spheroidal voids. Int. J. Plasticity, 24:1158-1189, 2008.

Leo Morin, Jean-Baptiste Leblond, and A. Amine Benzerga. Coalescence of voids by internal necking: Theoretical estimates and numerical results. Journal of the Mechanics and Physics of Solids, 75(0):140 - 158, 2015. ISSN 0022-5096. doi: http://dx.doi.org/10.1016/j.jmps.2014.11.009. URL http://www.sciencedirect.com/science/article/pii/S0022509614002257.

T Pardoen and J.W Hutchinson. An extended model for void growth and coalescence. Journal of the Mechanics and Physics of Solids, 48(12):2467 - 2512, 2000. ISSN 0022-5096. doi: http://dx.doi.org/10.1016/S0022-5096(00)00019-3. URL http://www.sciencedirect.com/science/article/pii/S0022509600000193.

J. Paux, L. Morin, R. Brenner, and D. Kondo. An approximate yield criterion for porous single crystals (in press). Eur. J. Mech. A/Solids, 51(1-10), 2015.

P. Ponte Castañeda. The effective mechanical properties of nonlinear isotropic composites. J. Mech. Phys. Solids, 35: 45-71, 1991a.

P. Ponte Castañeda. Effective properties in power-law creep. Mechanics of creep brittle materials, 2:218-229, 1991b.

P. Ponte Castañeda. Second-order homogenization estimates for nonlinear composites incorporating field fluctuations: I-theory. Journal of the Mechanics and Physics of Solids, 50:737-757, 2002a.

P. Ponte Castañeda. Second-order homogenization estimates for nonlinear composites incorporating field fluctuations: Ii-applications. Journal of the Mechanics and Physics of Solids, 50:759-782, 2002b.

P. Ponte Castañeda and J.R. Willis. The effect of spatial distribution on the effective behavior of composite materials and cracked media. J. Mech. Phys. Solids, 43:1919-1951, 1995.

B. Revil-Baudard and O. Cazacu. New three-dimensional strain-rate potentials for isotropic porous metals: Role of the plastic flow of the matrix. Int. J. Plasticity, 60:101-117, 2014.

J.R. Rice and D.M. Tracey. On the ductile enlargement of voids in triaxial stress fields. J. Mech. Phys. Solids, 17:201-217, 1969.

M.D. Rintoul and S. Torquato. Reconstruction of the structure of dispersions. J. Colloid Interface Sci., 186:467-476, 1997.

J. Segurado and J. Llorca. A numerical approximation to the elastic properties of sphere-reinforced composites. Journal of the Mechanics and Physics of Solids, 50:2107-2121, 2002.

J. Segurado and J. Llorca. Discrete dislocation dynamics analysis of the effect of lattice orientation on void growth in single crystals. Int. J. Plasticity, 26:806-819, 2010.

V.B. Shenoy, R. Phillips, and E.B. Tadmor. Nucleation of dislocations beneath a plane strain indenter. Journal of the Mechanics and Physics of Solids, 48:649-673, 2000.

V. Sorkin, R.S. Elliott, and E.B. Tadmor. A local quasicontinuum method for 3d multilattice crystalline materials: Application to shape-memory alloys. Modelling and Simulation in Materials Science and Engineering, 22(5):055001, 2014.

A. Srivastava and A. Needleman. Porosity evolution in a creeping single crystal. Model. Simul. Mater. Sci. Eng., $20,2012$.

A. Srivastava and A. Needleman. Effect of crystal orientation on porosity evolution in a creeping single crystal. Mechanics of Materials, (0):-, 2015. ISSN 0167-6636. doi: http://dx.doi.org/10.1016/j.mechmat.2015.01.015. URL http://www.sciencedirect.com/science/article/pii/S0167663615000228.

A. Srivastava, S. Gopagoni, A. Needleman, V. Seetharaman, A. Staroselsky, and R. Banerjee. Effect of specimen thickness on the creep response of a ni-based single-crystal superalloy. Acta Materialia, 60:5697-5711, 2012.

P. Suquet. Overall properties of nonlinear composites: a modified secant moduli theory and its link with ponte castañeda's nonlinear variational procedure. C. R. Acad. Sci. Paris II, 320:563-571, 1995.

T. Tang, S. Kim, and M. Horstemeyer. Fatigue crack growth in magnesium single crystals under cyclic loading: molecular dynamics simulation. Comput. Mater. Sci., 48:426-439, 2010a.

T. Tang, S. Kim, and M. Horstemeyer. Molecular dynamics simulations of void growth and coalescence in single crystal magnesium. Acta Mater., 58:4742-4759, 2010b.

S. Torquato. Random heterogeneous materials: Micorstructures and macroscopic properties. Springer, 2002.

S. Traiviratana, E. Bringa, D. Benson, and Meyers. Void growth in metals: atomistic calculations. Acta Mater., 56: 3874-3886, 2008.

V. Tvergaard and K.L. Nielsen. Relations between a micro-mechanical model and a damage model for ductile failure in shear. J. Mech. Phys. Solids, 58:1243-1252, 2010.

J.R. Willis. Bounds and self-consistent estimates for the overall properties of anisotropic composites. J. Mech. Phys. Solids, 25:185-202, 1977.

J.R. Willis. Variational principles and bounds for the overall properties of composites. Continuum Models and Discrete 
Systems, 2:185-212, 1978.

S.K. Yerra, C. Tekoglu, F. Scheyvaerts, L. Delannay, P. Van Houtte, and T. Pardoen. Void growth and coalescence in single crystals. Int. J. Solids Struct., 47:1016-1029, 2010.

K. Zhao, C. Chen, Y. Shen, and T. Lu. Molecular dynamics study on the nano-void growth in face-centered cubic single crystal copper. Comput. Mater. Sci., 46:749-754, 2009. 